\title{
A New Bayesian Approach to Robustness Against Outliers in Linear Regression - Supplementary Material
}

\author{
Philippe Gagnon*, Alain Desgagné ${ }^{\dagger}$ and Mylène Bédard ${ }^{\ddagger}$
}

Proposition 2.1 and Theorem 2.1 from our paper are proved in detail in Sections 1.1 and 1.2, respectively. In Section 2, we complete Section 3.2 of our paper regarding the claims about the divergence and the regularity conditions in Bunke et al. (1998). Finally, we provide a result in Section 3 that was used to verify that all point estimators of $\boldsymbol{\beta}$ under the normal model correspond to OLS, as mentioned in Section 3.3 in our paper.

\section{Proofs}

Recall the assumptions on $f: f$ is a strictly positive continuous $\mathrm{PDF}$ on $\mathbb{R}$ that is symmetric with respect to the origin, and such that both tails of $|z| f(z)$ are monotonic, which implies that the tails of $f(z)$ are also monotonic. The monotonicity of the tails of $f(z)$ and $|z| f(z)$ implies that there exists a constant $M>0$ such that

$$
|y| \geq|z| \geq M \Rightarrow f(y) \leq f(z) \text { and }|y| f(y) \leq|z| f(z) .
$$

All these assumptions on $f$ imply that $f(z)$ and $|z| f(z)$ are bounded on the real line, and both converge to 0 as $|z| \rightarrow \infty$. We can therefore define the constant $B>0$ as follows:

$$
B:=\max \left\{\sup _{z \in \mathbb{R}} f(z), \sup _{z \in \mathbb{R}}|z| f(z), \sup _{\boldsymbol{\beta} \in \mathbb{R}^{p}, \sigma>0} \pi(\boldsymbol{\beta}, \sigma) / \max (1,1 / \sigma)\right\} .
$$

\subsection{Proof of Proposition 2.1}

To prove that $\pi\left(\boldsymbol{\beta}, \sigma \mid \mathbf{y}_{\mathbf{n}}\right)$ is proper (the proof for $\pi\left(\boldsymbol{\beta}, \sigma \mid \mathbf{y}_{\mathbf{k}}\right)$ is omitted because it is similar), it suffices to show that the marginal $m\left(\mathbf{y}_{\mathbf{n}}\right)$ is finite. Recall that we require that $n>p+1$. The reader will notice that only $n \geq p+1$ is required if $\pi(\boldsymbol{\beta}, \sigma)$ is bounded by $B / \sigma$ for all $\sigma>0$ and $\boldsymbol{\beta} \in \mathbb{R}^{p}$ (instead of $\pi(\boldsymbol{\beta}, \sigma)$ is bounded by $B \max (1,1 / \sigma)$ ).

We first show that the function is integrable on the area where the ratio $1 / \sigma$ is bounded. More precisely, we consider $\boldsymbol{\beta} \in \mathbb{R}^{p}$ and $\delta M^{-1} \leq \sigma<\infty$, where $\delta$ is a positive

\footnotetext{
*Department of Statistics, University of Oxford, 24-29 St Giles', Oxford, OX1 3LB, United Kingdom. philippe.gagnon@stats.ox.ac.uk

†Département de mathématiques, Université du Québec à Montréal, C.P. 8888, Succursale Centreville, Montréal, QC, H3C 3P8, Canada. desgagne.alain@uqam.ca

${ }_{\ddagger}$ Département de mathématiques et de statistique, Université de Montréal, C.P. 6128, Succursale Centre-ville, Montréal, QC, H3C 3J7, Canada. bedard@dms.umontreal.ca
} 
constant that can be chosen as small as we want (upper bounds are provided in the proof). We next show that the function is integrable on the area where the ratio $1 / \sigma$ approaches infinity, that is $0<\sigma<\delta M^{-1}$. We have

$$
\begin{aligned}
& \int_{\delta M^{-1}}^{\infty} \int_{\mathbb{R}^{p}} \pi(\boldsymbol{\beta}, \sigma) \prod_{i=1}^{n}(1 / \sigma) f\left(\left(y_{i}-\mathbf{x}_{i}^{T} \boldsymbol{\beta}\right) / \sigma\right) d \boldsymbol{\beta} d \sigma \\
& \quad \stackrel{a}{\leq} B^{n-p+1} \int_{\delta M^{-1}}^{\infty} \max \left(1, \frac{1}{\sigma}\right) \frac{1}{\sigma^{n-p}} \int_{\mathbb{R}^{p}} \prod_{i=1}^{p} \frac{1}{\sigma} f\left(\frac{y_{i}-\mathbf{x}_{i}^{T} \boldsymbol{\beta}}{\sigma}\right) d \boldsymbol{\beta} d \sigma \\
& \quad \stackrel{b}{\leq} \max \left(1, \frac{M}{\delta}\right) B^{n-p+1}\left|\operatorname{det}\left(\begin{array}{c}
\mathbf{x}_{1}^{T} \\
\vdots \\
\mathbf{x}_{p}^{T}
\end{array}\right)\right|^{-1} \int_{\delta M^{-1}}^{\infty} \frac{1}{\sigma^{n-p}} d \sigma \prod_{i=1}^{p} \int_{-\infty}^{\infty} f\left(u_{i}\right) d u_{i} \\
& \quad \propto \int_{\delta M^{-1}}^{\infty} \frac{1}{\sigma^{n-p}} d \sigma \stackrel{c}{=}(M / \delta)^{n-p-1} /(n-p-1)<\infty .
\end{aligned}
$$

In Step $a$, we use $\pi(\boldsymbol{\beta}, \sigma) \leq B \max (1,1 / \sigma)$ and we bound each of $n-p$ densities $f$ by $B$. In Step $b$, we use the change of variables $u_{i}=\left(y_{i}-\mathbf{x}_{i}^{T} \boldsymbol{\beta}\right) / \sigma$ for $i=1, \ldots, p$. The determinant is non-null because all explanatory variables are continuous. Indeed, consider the case $p=2$ for instance (i.e. the simple linear regression); the determinant is different from 0 provided that $x_{12} \neq x_{22}$, and this happens with probability 1 . When any type of explanatory variables is considered, we need to be able to select $p$ observations, say those with $\mathbf{x}_{i_{1}}, \ldots, \mathbf{x}_{i_{p}}$, such that the matrix with rows $\mathbf{x}_{i_{1}}^{T}, \ldots, \mathbf{x}_{i_{p}}^{T}$ has a non-null determinant. This is possible when the design matrix has full rank, which is specified in Remark 2.1 in our paper. In Step $c$, we use $n>p+1$. Note that if, instead, we bound $\pi(\boldsymbol{\beta}, \sigma)$ by $B / \sigma$ in Step $a$, one can verify that the condition $n \geq p+1$ is sufficient to bound above the integral.

We now show that the integral is finite on $\boldsymbol{\beta} \in \mathbb{R}^{p}$ and $0<\sigma<\delta M^{-1}$. In this area, the ratio $(1 / \sigma)$ approaches infinity. We have to carefully analyse the subareas where $y_{i}-\mathbf{x}_{i}^{T} \boldsymbol{\beta}$ is close to 0 in order to deal with the $0 / 0$ form of the ratios $\left(y_{i}-\mathbf{x}_{i}^{T} \boldsymbol{\beta}\right) / \sigma$. In order to achieve this, we split the domain of $\boldsymbol{\beta}$ as follows:

$$
\begin{aligned}
\mathbb{R}^{p}=[ & \left.\cap_{i_{1}=1}^{n} \mathcal{R}_{i_{1}}^{c}\right] \cup\left[\cup_{i_{1}=1}^{n}\left(\mathcal{R}_{i_{1}} \cap\left(\cap_{i_{2}=1\left(i_{2} \neq i_{1}\right)}^{n} \mathcal{R}_{i_{2}}^{c}\right)\right)\right] \\
& \cup\left[\cup_{i_{1}, i_{2}=1\left(i_{1} \neq i_{2}\right)}^{n}\left(\mathcal{R}_{i_{1}} \cap \mathcal{R}_{i_{2}} \cap\left(\cap_{i_{3}=1\left(i_{3} \neq i_{1}, i_{2}\right)}^{n} \mathcal{R}_{i_{3}}^{c}\right)\right)\right] \\
& \cup \cdots \cup\left[\cup_{i_{1}, i_{2}, \ldots, i_{p}=1\left(i_{j} \neq i_{s} \forall i_{j}, i_{s} \text { s.t. } j \neq s\right)}\left(\mathcal{R}_{i_{1}} \cap \mathcal{R}_{i_{2}} \cap \ldots \cap \mathcal{R}_{i_{p}}\right)\right],
\end{aligned}
$$

where $\mathcal{R}_{i}:=\left\{\boldsymbol{\beta}:\left|y_{i}-\mathbf{x}_{i}^{T} \boldsymbol{\beta}\right|<\delta\right\}, i \in\{1, \ldots, n\}$. The set $\mathcal{R}_{i}$ represents the hyperplanes $y=\mathbf{x}_{i}^{T} \boldsymbol{\beta}$ characterised by the different values of $\boldsymbol{\beta}$ that satisfy $\left|y_{i}-\mathbf{x}_{i}^{T} \boldsymbol{\beta}\right|<\delta$. In other words, it represents the hyperplanes passing near the point $\left(\mathbf{x}_{i}, y_{i}\right)$, and more precisely, at a vertical distance of less than $\delta$. The set $\cap_{i_{1}=1}^{n} \mathcal{R}_{i_{1}}^{c}$ is therefore comprised of the hyperplanes that are not passing close to any point. The set $\cup_{i_{1}=1}^{n}\left(\mathcal{R}_{i_{1}} \cap\right.$ $\left.\left(\cap_{i_{2}=1\left(i_{2} \neq i_{1}\right)}^{n} \mathcal{R}_{i_{2}}^{c}\right)\right)$ represents the hyperplanes passing near one (and only one) point. The set $\cup_{i_{1}, i_{2}=1\left(i_{1} \neq i_{2}\right)}^{n}\left(\mathcal{R}_{i_{1}} \cap \mathcal{R}_{i_{2}} \cap\left(\cap_{i_{3}=1\left(i_{3} \neq i_{1}, i_{2}\right)}^{n} \mathcal{R}_{i_{3}}^{c}\right)\right)$ represents the hyperplanes passing near two (and only two) points, and so on. 
We choose $\delta$ small enough to ensure that $\mathcal{R}_{i_{1}} \cap \mathcal{R}_{i_{2}} \cap \ldots \cap \mathcal{R}_{i_{p}} \cap \mathcal{R}_{i_{p+1}}=\varnothing$ when $i_{1}, \ldots, i_{p+1}$ are all different. It is possible to do so because an hyperplane passes through no more than $p$ points. This implies that

$$
\begin{aligned}
{\left[\cup_{i_{1}, i_{2}, \ldots, i_{p}=1\left(i_{j} \neq i_{s} \forall i_{j}, i_{s} \text { s.t. } j \neq s\right)}^{n}\left(\mathcal{R}_{i_{1}} \cap \mathcal{R}_{i_{2}} \cap \ldots \cap \mathcal{R}_{i_{p}}\right)\right] } \\
=\left[\cup _ { i _ { 1 } , i _ { 2 } , \ldots , i _ { p } = 1 ( i _ { j } \neq i _ { s } \forall i _ { j } , i _ { s } \text { s.t. } j \neq s ) } \left(\mathcal{R}_{i_{1}} \cap \mathcal{R}_{i_{2}} \cap \ldots \cap \mathcal{R}_{i_{p}}\right.\right. \\
\left.\left.\cap\left(\cap_{i_{p+1}=1\left(i_{p+1} \neq i_{1}, i_{2}, \ldots, i_{p}\right)}^{n} \mathcal{R}_{i_{p+1}}^{c}\right)\right)\right] .
\end{aligned}
$$

Note that all sets $\mathcal{R}_{i_{1}} \cap \mathcal{R}_{i_{2}} \cap \ldots \cap \mathcal{R}_{i_{p}}$ are nonempty when $i_{1}, \ldots, i_{p}$ are all different, because all explanatory variables are continuous (which implies that the $p \times p$ matrix with rows given by $\mathbf{x}_{i_{1}}^{T}, \ldots, \mathbf{x}_{i_{p}}^{T}$ has a determinant different from 0 ). As mentioned above, if they are not all continuous, we have to select them in order to have to have a matrix with a non-null determinant. Note also that $\mathcal{R}_{i_{1}} \cap\left(\cap_{i_{2}=1\left(i_{2} \neq i_{1}\right)}^{n} \mathcal{R}_{i_{2}}^{c}\right)$ is nonempty for all $i_{1}, \mathcal{R}_{i_{1}} \cap \mathcal{R}_{i_{2}} \cap\left(\cap_{i_{3}=1\left(i_{3} \neq i_{1}, i_{2}\right)}^{n} \mathcal{R}_{i_{3}}^{c}\right)$ is nonempty for all $i_{1}, i_{2}$ such that $i_{1} \neq i_{2}$, and so on. Finally note that the decomposition of $\mathbb{R}^{p}$ given in (1.2) is comprised of $\sum_{i=0}^{p}\left(\begin{array}{c}n \\ i\end{array}\right)$ mutually exclusive sets given by $\cap_{i_{1}=1}^{n} \mathcal{R}_{i_{1}}^{c}, \mathcal{R}_{i_{1}} \cap\left(\cap_{i_{2}=1\left(i_{2} \neq i_{1}\right)}^{n} \mathcal{R}_{i_{2}}^{c}\right), i_{1}=1, \ldots, n, \mathcal{R}_{i_{1}} \cap$ $\mathcal{R}_{i_{2}} \cap\left(\cap_{i_{3}=1\left(i_{3} \neq i_{1}, i_{2}\right)}^{n} \mathcal{R}_{i_{3}}^{c}\right), i_{1}, i_{2}=1, \ldots, n$ with $i_{1} \neq i_{2}$, and so on.

We now consider $0<\sigma<\delta M^{-1}$ and $\boldsymbol{\beta}$ in one of the $\sum_{i=0}^{p}\left(\begin{array}{c}n \\ i\end{array}\right)$ mutually exclusive sets given in (1.2). As explained above, the difficulty lies in dealing with the hyperplanes $\boldsymbol{\beta}$ that are such that $\left|y_{i}-\mathbf{x}_{i}^{T} \boldsymbol{\beta}\right|<\delta$ for some points $\left(\mathbf{x}_{i}, y_{i}\right)$. The strategy is essentially to use the product of $(1 / \sigma) f\left(\left(y_{i}-\mathbf{x}_{i}^{T} \boldsymbol{\beta}\right) / \sigma\right)$ of these points to integrate over $\boldsymbol{\beta}$, and to bound the other terms of $m\left(\mathbf{y}_{\mathbf{n}}\right)$. Therefore, if $\boldsymbol{\beta} \in \mathcal{R}_{i_{1}} \cap \mathcal{R}_{i_{2}} \cap \ldots \cap \mathcal{R}_{i_{p}}$, we consider the points $\left(\mathbf{x}_{i_{1}}, y_{i_{1}}\right),\left(\mathbf{x}_{i_{2}}, y_{i_{2}}\right), \ldots,\left(\mathbf{x}_{i_{p}}, y_{i_{p}}\right)$ to integrate over $\boldsymbol{\beta}$. If $\boldsymbol{\beta} \in \mathcal{R}_{i_{1}} \cap \mathcal{R}_{i_{2}} \cap \ldots \mathcal{R}_{i_{p-1}} \cap$ $\left(\cap_{i_{p}=1\left(i_{p} \neq i_{1}, \ldots, i_{p-1}\right)}^{n} \mathcal{R}_{i_{p}}^{c}\right)$, we consider the points $\left(\mathbf{x}_{i_{1}}, y_{i_{1}}\right),\left(\mathbf{x}_{i_{2}}, y_{i_{2}}\right), \ldots,\left(\mathbf{x}_{i_{p-1}}, y_{i_{p-1}}\right)$, and any other point $\left(\mathbf{x}_{i_{p}}, y_{i_{p}}\right)$ (leading to a matrix with a non-null determinant) to integrate over $\boldsymbol{\beta}$, and so on. We have

$$
\begin{aligned}
& \pi(\boldsymbol{\beta}, \sigma) \prod_{i=1}^{n}(1 / \sigma) f\left(\left(y_{i}-\mathbf{x}_{i}^{T} \boldsymbol{\beta}\right) / \sigma\right) \stackrel{a}{\leq}(B / \sigma) \max \left(\delta M^{-1}, 1\right) \prod_{i=1}^{n}(1 / \sigma) f\left(\left(y_{i}-\mathbf{x}_{i}^{T} \boldsymbol{\beta}\right) / \sigma\right) \\
& \propto(1 / \sigma) \prod_{i \in\left\{i_{1}, \ldots, i_{p}\right\}}(1 / \sigma) f\left(\left(y_{i}-\mathbf{x}_{i}^{T} \boldsymbol{\beta}\right) / \sigma\right) \prod_{i \notin\left\{i_{1}, \ldots, i_{p}\right\}}(1 / \sigma) f\left(\left(y_{i}-\mathbf{x}_{i}^{T} \boldsymbol{\beta}\right) / \sigma\right) \\
& \stackrel{b}{\leq}(1 / \sigma)[(1 / \sigma) f(\delta / \sigma)]^{n-p} \prod_{i \in\left\{i_{1}, \ldots, i_{p}\right\}}(1 / \sigma) f\left(\left(y_{i}-\mathbf{x}_{i}^{T} \boldsymbol{\beta}\right) / \sigma\right) \\
& \stackrel{c}{\leq}[B / \delta]^{n-p-1}\left(1 / \sigma^{2}\right) f(\delta / \sigma) \prod_{i \in\left\{i_{1}, \ldots, i_{p}\right\}}(1 / \sigma) f\left(\left(y_{i}-\mathbf{x}_{i}^{T} \boldsymbol{\beta}\right) / \sigma\right) .
\end{aligned}
$$

In Step $a$, we use $\pi(\boldsymbol{\beta}, \sigma) \leq B \max (1,1 / \sigma)=(B / \sigma) \max (\sigma, 1) \leq(B / \sigma) \max \left(\delta M^{-1}, 1\right)$. In Step $b$, for all $i \notin\left\{i_{1}, \ldots, i_{p}\right\}$ we use $f\left(\left(y_{i}-\mathbf{x}_{i}^{T} \boldsymbol{\beta}\right) / \sigma\right) \leq f(\delta / \sigma)$ by the monotonicity of the tails of $f$ because $\left|y_{i}-\mathbf{x}_{i}^{T} \boldsymbol{\beta}\right| / \sigma \geq \delta / \sigma \geq \delta \delta^{-1} M=M$. In Step $c$, we bound $n-p-1$ terms $(1 / \sigma) f(\delta / \sigma)$ by $B / \delta$. 
Finally, we bound the integral of $\left(1 / \sigma^{2}\right) f(\delta / \sigma) \prod_{i \in\left\{i_{1}, \ldots, i_{p}\right\}}(1 / \sigma) f\left(\left(y_{i}-\mathbf{x}_{i}^{T} \boldsymbol{\beta}\right) / \sigma\right)$ by

$$
\begin{aligned}
& \int_{0}^{\infty}\left(1 / \sigma^{2}\right) f(\delta / \sigma) \int_{\mathbb{R}^{p}} \prod_{i \in\left\{i_{1}, \ldots, i_{p}\right\}}(1 / \sigma) f\left(\left(y_{i}-\mathbf{x}_{i}^{T} \boldsymbol{\beta}\right) / \sigma\right) d \boldsymbol{\beta} d \sigma \\
& \stackrel{a}{=}\left|\operatorname{det}\left(\begin{array}{c}
\mathbf{x}_{i_{1}}^{T} \\
\vdots \\
\mathbf{x}_{i_{p}}^{T}
\end{array}\right)\right|{ }^{-1}\left(1 / \sigma^{2}\right) f(\delta / \sigma) d \sigma \stackrel{b}{=}\left|\operatorname{det}\left(\begin{array}{c}
\mathbf{x}_{i_{1}}^{T} \\
\vdots \\
\mathbf{x}_{i_{p}}^{T}
\end{array}\right)\right|^{-1} \int_{0}^{\infty} f\left(\sigma^{\prime}\right) d \sigma^{\prime}<\infty
\end{aligned}
$$

In Step $a$, we use the same change of variables as above: $u_{j}=\left(y_{i_{j}}-\mathbf{x}_{i_{j}}^{T} \boldsymbol{\beta}\right) / \sigma$ for $j=1, \ldots, p$. In Step $b$, we use the change of variable $\sigma^{\prime}=\delta / \sigma$.

We now prove that the $M$-moments exist if $n>p+1+M$ (considering the whole data set, the proof for the posterior expectations based on the nonoutliers only is omitted because it is similar). It is easy to prove that $\mathbb{E}\left[\sigma^{M} \mid \mathbf{y}_{\mathbf{n}}\right]<\infty$, from what has been demonstrated above. Indeed,

$$
\begin{aligned}
\mathbb{E}\left[\sigma^{M} \mid \mathbf{y}_{\mathbf{n}}\right] & =\left[m\left(\mathbf{y}_{\mathbf{n}}\right)\right]^{-1} \int_{0}^{\infty} \int_{\mathbb{R}^{p}} \sigma^{M} \pi(\boldsymbol{\beta}, \sigma) \prod_{i=1}^{n}(1 / \sigma) f\left(\left(y_{i}-\mathbf{x}_{i}^{T} \boldsymbol{\beta}\right) / \sigma\right) d \boldsymbol{\beta} d \sigma \\
& \leq B^{M}\left[m\left(\mathbf{y}_{\mathbf{n}}\right)\right]^{-1} \int_{0}^{\infty} \int_{\mathbb{R}^{p}} \pi(\boldsymbol{\beta}, \sigma) \prod_{i=M+1}^{n}(1 / \sigma) f\left(\left(y_{i}-\mathbf{x}_{i}^{T} \boldsymbol{\beta}\right) / \sigma\right) d \boldsymbol{\beta} d \sigma
\end{aligned}
$$

where $M$ densities $f$ have been bounded by $B$. We know that $m\left(\mathbf{y}_{\mathbf{n}}\right)$ is finite because $n>p+1$. We also know that the last integral above is finite because it corresponds to the marginal of $n-M$ observations, which is finite given that $n-M>p+1$.

For the expectations $\mathbb{E}\left[\left|\beta_{j}\right|^{M} \mid \mathbf{y}_{\mathbf{n}}\right]$, we detail the proof for the cases $M=1$ and $M=2$. From these, it will be clear that the result holds in general, with further technicalities. For the proof, we use that $\beta_{j}$ can be rewritten as $\mathbf{e}_{j}^{T} \boldsymbol{\beta}$, where $\mathbf{e}_{j}$ is a vector of size $p$ having 1 at the $j$-th position and 0's elsewhere. This vector can be expressed as a linear combination of $p$ vectors $\mathbf{x}_{i_{1}}, \ldots, \mathbf{x}_{i_{p}}, i_{1}, \ldots, i_{p} \in\{1, \ldots, n\}$, because these vectors are linearly independent and form a basis of $\mathbb{R}^{p}$ (given that all explanatory variables are continuous). Using the triangle inequality, we have

$$
\begin{array}{r}
\mathbb{E}\left[\left|\beta_{j}\right| \mid \mathbf{y}_{\mathbf{n}}\right]=\mathbb{E}\left[\left|\mathbf{e}_{j}^{T} \boldsymbol{\beta}\right| \mid \mathbf{y}_{\mathbf{n}}\right]=\mathbb{E}\left[\left|\sum_{s=1}^{p} a_{s} \mathbf{x}_{i_{s}}^{T} \boldsymbol{\beta}\right| \mid \mathbf{y}_{\mathbf{n}}\right] \leq \sum_{s=1}^{p}\left|a_{s}\right| \mathbb{E}\left[\left|\mathbf{x}_{i_{s}}^{T} \boldsymbol{\beta}\right| \mid \mathbf{y}_{\mathbf{n}}\right] \\
\leq \sum_{s=1}^{p}\left|a_{s}\right|\left(\left|y_{i_{s}}\right|+\mathbb{E}\left[\left|y_{i_{s}}-\mathbf{x}_{i_{s}}^{T} \boldsymbol{\beta}\right| \mid \mathbf{y}_{\mathbf{n}}\right]\right)
\end{array}
$$

where $a_{1}, \ldots, a_{p} \in \mathbb{R}$. We can prove that $\mathbb{E}\left[\left|y_{i_{s}}-\mathbf{x}_{i_{s}}^{T} \boldsymbol{\beta}\right| \mid \mathbf{y}_{\mathbf{n}}\right]<\infty$ in the same way that we proved that $\mathbb{E}\left[\sigma^{M} \mid \mathbf{y}_{\mathbf{n}}\right]<\infty$, using instead that

$$
\left(\left|y_{i_{s}}-\mathbf{x}_{i_{s}}^{T} \boldsymbol{\beta}\right| / \sigma\right) f\left(\left(y_{i_{s}}-\mathbf{x}_{i_{s}}^{T} \boldsymbol{\beta}\right) / \sigma\right) \leq B
$$


Therefore, $\mathbb{E}\left[\left|\beta_{j}\right| \mid \mathbf{y}_{\mathbf{n}}\right]<\infty$ if $n>p+2$. For the second moment, using again the triangle inequality we have

$$
\begin{aligned}
\mathbb{E}\left[\left|\beta_{j}^{2}\right| \mid \mathbf{y}_{\mathbf{n}}\right] & =\mathbb{E}\left[\left|\left(\sum_{s=1}^{p} a_{s} \mathbf{x}_{i_{s}}^{T} \boldsymbol{\beta}\right)^{2}\right| \mid \mathbf{y}_{\mathbf{n}}\right] \\
& =\mathbb{E}\left[\left|\sum_{s=1}^{p}\left(a_{s} \mathbf{x}_{i_{s}}^{T} \boldsymbol{\beta}\right)^{2}+\sum_{s, t(s \neq t)} a_{s} \mathbf{x}_{i_{s}}^{T} \boldsymbol{\beta} a_{t} \mathbf{x}_{i_{t}}^{T} \boldsymbol{\beta}\right| \mid \mathbf{y}_{\mathbf{n}}\right] \\
& \leq \mathbb{E}\left[\sum_{s=1}^{p}\left(a_{s} \mathbf{x}_{i_{s}}^{T} \boldsymbol{\beta}\right)^{2} \mid \mathbf{y}_{\mathbf{n}}\right]+\mathbb{E}\left[\left|\sum_{s, t(s \neq t)} a_{s} \mathbf{x}_{i_{s}}^{T} \boldsymbol{\beta} a_{t} \mathbf{x}_{i_{t}}^{T} \boldsymbol{\beta}\right| \mid \mathbf{y}_{\mathbf{n}}\right] .
\end{aligned}
$$

We analyse the two last terms separately, starting with the second one. Using again the triangle inequality we have,

$$
\begin{gathered}
\mathbb{E}\left[\left|\sum_{s, t(s \neq t)} a_{s} \mathbf{x}_{i_{s}}^{T} \boldsymbol{\beta} a_{t} \mathbf{x}_{i_{t}}^{T} \boldsymbol{\beta}\right| \mid \mathbf{y}_{\mathbf{n}}\right] \leq \sum_{s, t(s \neq t)}\left|a_{s} a_{t}\right| \mathbb{E}\left[\left|\mathbf{x}_{i_{s}}^{T} \boldsymbol{\beta}\right|\left|\mathbf{x}_{i_{t}}^{T} \boldsymbol{\beta}\right| \mid \mathbf{y}_{\mathbf{n}}\right] \\
\leq \sum_{s, t(s \neq t)}\left|a_{s} a_{t}\right|\left(\mathbb{E}\left[\left|y_{i_{s}}-\mathbf{x}_{i_{s}}^{T} \boldsymbol{\beta}\right|\left|y_{i_{t}}-\mathbf{x}_{i_{t}}^{T} \boldsymbol{\beta}\right| \mid \mathbf{y}_{\mathbf{n}}\right]+\left|y_{i_{s}}\right| \mathbb{E}\left[\left|y_{i_{t}}-\mathbf{x}_{i_{t}}^{T} \boldsymbol{\beta}\right| \mid \mathbf{y}_{\mathbf{n}}\right]\right. \\
\left.+\left|y_{i_{t}}\right| \mathbb{E}\left[\left|y_{i_{s}}-\mathbf{x}_{i_{s}}^{T} \boldsymbol{\beta}\right| \mid \mathbf{y}_{\mathbf{n}}\right]+\left|y_{i_{s}} y_{i_{t}}\right|\right)
\end{gathered}
$$

All the terms in the sum are finite if $n>p+3$. Also,

$$
\begin{aligned}
\mathbb{E}\left[\sum_{s=1}^{p}\left(a_{s} \mathbf{x}_{i_{s}}^{T} \boldsymbol{\beta}\right)^{2} \mid \mathbf{y}_{\mathbf{n}}\right]=\sum_{s=1}^{p} a_{s}^{2} \mathbb{E}\left[\left|\mathbf{x}_{i_{s}}^{T} \boldsymbol{\beta} \mathbf{x}_{i_{s}}^{T} \boldsymbol{\beta}\right| \mid \mathbf{y}_{\mathbf{n}}\right] & =\sum_{s=1}^{p} a_{s}^{2} \mathbb{E}\left[\left|\sum_{t=1}^{p} b_{t} \mathbf{x}_{i_{t}}^{T} \boldsymbol{\beta} \mathbf{x}_{i_{s}}^{T} \boldsymbol{\beta}\right| \mid \mathbf{y}_{\mathbf{n}}\right] \\
& \leq \sum_{s=1}^{p} a_{s}^{2} \sum_{t=1}^{p} b_{t} \mathbb{E}\left[\left|\mathbf{x}_{i_{t}}^{T} \boldsymbol{\beta} \mathbf{x}_{i_{s}}^{T} \boldsymbol{\beta}\right| \mid \mathbf{y}_{\mathbf{n}}\right]
\end{aligned}
$$

and we proceed as before. In the second equality, we write $\mathbf{x}_{i_{s}}$ as a linear combination of $\mathbf{x}_{i_{1}}, \ldots, \mathbf{x}_{i_{p}}, i_{1}, \ldots, i_{p} \in\{1, \ldots, n\} \backslash\left\{i_{s}\right\}$. To be able to do this, we need to select $p$ linearly independent vectors among the remaining $n-1 \geq p$. It is possible given that $n>p+3$ and all explanatory variables are continuous. Therefore, $\mathbb{E}\left[\left|\beta_{j}\right|^{2} \mid \mathbf{y}_{\mathbf{n}}\right]<\infty$ if $n>p+3$.

\subsection{Proof of Theorem 2.1}

Recall that we assume that $\ell \leq n / 2-(p-1 / 2) \Leftrightarrow k \geq n / 2+(p-1 / 2) \Leftrightarrow k \geq \ell+2 p-1$. In addition, we will assume that $\ell \geq 1$, i.e. that there is at least one outlier, otherwise the proof is trivial. A proposition and a lemma that are used in the proof are first given, and the proofs of Results (a) to (d) follow. The proofs of this proposition and this lemma can be found in Desgagné (2015). 
Proposition 1.1 (Dominance). If $s \in L_{0}(\infty)$ and $g \in L_{\rho}(\infty)$, then for all $\delta>0$, there exists a constant $A(\delta)>1$ such that $z \geq A(\delta)$ implies that

$$
(\log z)^{-\delta}<s(z)<(\log z)^{\delta} \quad \text { and } \quad(\log z)^{-\rho-\delta}<g(z)<(\log z)^{-\rho+\delta} .
$$

Lemma 1.1. For all $\lambda \geq 0, \forall \tau \geq 1$, there exists a constant $D(\lambda, \tau) \geq 1$ such that $z \in \mathbb{R}$ and $(\mu, \sigma) \in[-\lambda, \lambda] \times[1 / \tau, \tau]$ implies that

$$
1 / D(\lambda, \tau) \leq(1 / \sigma) f((z-\mu) / \sigma) / f(z) \leq D(\lambda, \tau)
$$

Note that Lemma 1.1 is a corollary of Proposition 2.2 in our paper.

Proof of Result (a). We first observe that

$$
\begin{aligned}
\frac{m\left(\mathbf{y}_{\mathbf{n}}\right)}{m\left(\mathbf{y}_{\mathbf{k}}\right) \prod_{i=1}^{n}\left[f\left(y_{i}\right)\right]^{\ell_{i}}} & =\frac{m\left(\mathbf{y}_{\mathbf{n}}\right)}{m\left(\mathbf{y}_{\mathbf{k}}\right) \prod_{i=1}^{n}\left[f\left(y_{i}\right)\right]^{\ell_{i}}} \int_{\mathbb{R}^{p}} \int_{0}^{\infty} \pi\left(\boldsymbol{\beta}, \sigma \mid \mathbf{y}_{\mathbf{n}}\right) d \sigma d \boldsymbol{\beta} \\
& =\int_{\mathbb{R}^{p}} \int_{0}^{\infty} \frac{\pi(\boldsymbol{\beta}, \sigma) \prod_{i=1}^{n}\left[(1 / \sigma) f\left(\left(y_{i}-\mathbf{x}_{i}^{T} \boldsymbol{\beta}\right) / \sigma\right)\right]^{k_{i}+\ell_{i}}}{m\left(\mathbf{y}_{\mathbf{k}}\right) \prod_{i=1}^{n}\left[f\left(y_{i}\right)\right]^{\ell_{i}}} d \sigma d \boldsymbol{\beta} \\
& =\int_{\mathbb{R}^{p}} \int_{0}^{\infty} \pi\left(\boldsymbol{\beta}, \sigma \mid \mathbf{y}_{\mathbf{k}}\right) \prod_{i=1}^{n}\left[\frac{(1 / \sigma) f\left(\left(y_{i}-\mathbf{x}_{i}^{T} \boldsymbol{\beta}\right) / \sigma\right)}{f\left(y_{i}\right)}\right]^{\ell_{i}} d \sigma d \boldsymbol{\beta} .
\end{aligned}
$$

We show that the last integral converges towards 1 as $\omega \rightarrow \infty$ to prove Result (a). If we use Lebesgue's dominated convergence theorem to interchange the limit $\omega \rightarrow \infty$ and the integral, we have

$$
\begin{aligned}
\lim _{\omega \rightarrow \infty} & \int_{\mathbb{R}^{p}} \int_{0}^{\infty} \pi\left(\boldsymbol{\beta}, \sigma \mid \mathbf{y}_{\mathbf{k}}\right) \prod_{i=1}^{n}\left[\frac{(1 / \sigma) f\left(\left(y_{i}-\mathbf{x}_{i}^{T} \boldsymbol{\beta}\right) / \sigma\right)}{f\left(y_{i}\right)}\right]^{\ell_{i}} d \sigma d \boldsymbol{\beta} \\
& =\int_{\mathbb{R}^{p}} \int_{0}^{\infty} \lim _{\omega \rightarrow \infty} \pi\left(\boldsymbol{\beta}, \sigma \mid \mathbf{y}_{\mathbf{k}}\right) \prod_{i=1}^{n}\left[\frac{(1 / \sigma) f\left(\left(y_{i}-\mathbf{x}_{i}^{T} \boldsymbol{\beta}\right) / \sigma\right)}{f\left(y_{i}\right)}\right]^{\ell_{i}} d \sigma d \boldsymbol{\beta} \\
& =\int_{\mathbb{R}^{p}} \int_{0}^{\infty} \pi\left(\boldsymbol{\beta}, \sigma \mid \mathbf{y}_{\mathbf{k}}\right) d \sigma d \boldsymbol{\beta}=1,
\end{aligned}
$$

using Proposition 2.2 in our paper in the second equality, since $\mathbf{x}_{1}, \ldots, \mathbf{x}_{n}$ are fixed, and then Proposition 2.1 in our paper. Note that the conditions of Proposition 2.1 are satisfied because $k \geq \ell+2 p-1 \Rightarrow k \geq p+2$ (because $\ell \geq 1$ and $p \geq 2$ ). When any type of explanatory variables is considered, we select $p$ observations, say those with $\mathbf{x}_{i_{1}}, \ldots, \mathbf{x}_{i_{p}}$, such that the matrix with rows $\mathbf{x}_{i_{1}}^{T}, \ldots, \mathbf{x}_{i_{p}}^{T}$ has a non-null determinant. This is possible given the assumption mentioned in Remark 2.2 in our paper. Note also that pointwise convergence is sufficient, for any value of $\boldsymbol{\beta} \in \mathbb{R}^{p}$ and $\sigma>0$, once the limit is inside the integral. However, in order to use Lebesgue's dominated convergence theorem, we need to prove that the integrand is bounded by an integrable function of $\boldsymbol{\beta}$ and $\sigma$ that does not depend on $\omega$, for any value of $\omega \geq \mathrm{y}$, where $\mathrm{y}$ is a constant. The constant y can be chosen as large as we want, and minimum values for y will be 
given throughout the proof. In order to bound the integrand, we divide the domain of integration into two areas: $1 \leq \sigma<\infty$ and $0<\sigma<1$. Again, we want to separately analyse the area where the ratio $1 / \sigma$ approaches infinity.

We assumed that $y_{i}$ can be written as $y_{i}=a_{i}+b_{i} \omega$, where $\omega \rightarrow \infty$, and $a_{i}$ and $b_{i}$ are constants such that $a_{i} \in \mathbb{R}$ and $b_{i} \neq 0$ if $\ell_{i}=1$ (if the observation is an outlier). Therefore, the ranking of the elements in the set $\left\{\left|y_{i}\right|: \ell_{i}=1\right\}$ is primarily determined by the values $\left|b_{1}\right|, \ldots,\left|b_{n}\right|$, and we can choose the constant y larger than a certain threshold to ensure that this ranking remains unchanged for all $\omega \geq \mathrm{y}$. Without loss of generality, we assume for convenience that

$$
\omega=\min _{\left\{i: \ell_{i}=1\right\}}\left|y_{i}\right| \quad \text { and consequently } \quad \min _{\left\{i: \ell_{i}=1\right\}}\left|b_{i}\right|=1 .
$$

We now bound above the integrand on the first area.

Area 1: Consider $1 \leq \sigma<\infty$ and assume without loss of generality that $y_{1}, \ldots$, $y_{\ell+2 p-1}$ are $\ell+2 p-1$ nonoutliers (therefore $k_{1}=\ldots=k_{\ell+2 p-1}=1$ ). We have

$$
\begin{aligned}
& \pi\left(\boldsymbol{\beta}, \sigma \mid \mathbf{y}_{\mathbf{k}}\right) \prod_{i=1}^{n}\left[\frac{(1 / \sigma) f\left(\left(y_{i}-\mathbf{x}_{i}^{T} \boldsymbol{\beta}\right) / \sigma\right)}{f\left(y_{i}\right)}\right]^{\ell_{i}} \propto \frac{\pi(\boldsymbol{\beta}, \sigma)}{\sigma^{n}} \prod_{i=1}^{n} \frac{f\left(\left(y_{i}-\mathbf{x}_{i}^{T} \boldsymbol{\beta}\right) / \sigma\right)}{\left[f\left(y_{i}\right)\right]^{\ell_{i}}} \\
& \quad \stackrel{a}{\leq} \frac{B}{\sigma^{n}} \prod_{i=1}^{n} \frac{D\left(\left|a_{i}\right|, 1\right) f\left(\left(b_{i} \omega-\mathbf{x}_{i}^{T} \boldsymbol{\beta}\right) / \sigma\right)}{\left[f\left(y_{i}\right)\right]^{\ell_{i}}} \\
& \quad \stackrel{b}{\leq} \frac{1}{[f(\omega)]^{\ell}} \frac{B}{\sigma^{n}} \prod_{i=1}^{n} D\left(\left|a_{i}\right|, 1\right) f\left(\left(b_{i} \omega-\mathbf{x}_{i}^{T} \boldsymbol{\beta}\right) / \sigma\right)\left[\left|b_{i}\right| D\left(\left|a_{i}\right|,\left|b_{i}\right|\right)\right]^{\ell_{i}} \\
& \propto \frac{1}{[f(\omega)]^{\ell}} \frac{1}{\sigma^{n}} \prod_{i=1}^{n} f\left(\left(b_{i} \omega-\mathbf{x}_{i}^{T} \boldsymbol{\beta}\right) / \sigma\right) \\
& \stackrel{c}{=} \frac{1}{[f(\omega)]^{\ell}} \frac{1}{\sigma^{n}} \prod_{i=1}^{n}\left[f\left(\mathbf{x}_{i}^{T} \boldsymbol{\beta} / \sigma\right)\right]^{k_{i}}\left[f\left(\left(b_{i} \omega-\mathbf{x}_{i}^{T} \boldsymbol{\beta}\right) / \sigma\right)\right]^{\ell_{i}} \\
& \stackrel{d}{=} \frac{\prod_{i=1}^{p}(1 / \sigma) f\left(\mathbf{x}_{i}^{T} \boldsymbol{\beta} / \sigma\right)}{\sigma^{k-p-1 / 2}}\left[\frac{\omega / \sigma}{\omega f(\omega)}\right]^{\ell} \frac{1}{\sigma^{1 / 2}} \prod_{i=p+1}^{n}\left[f\left(\mathbf{x}_{i}^{T} \boldsymbol{\beta} / \sigma\right)\right]^{k_{i}}\left[f\left(\left(b_{i} \omega-\mathbf{x}_{i}^{T} \boldsymbol{\beta}\right) / \sigma\right)\right]^{\ell_{i}} .
\end{aligned}
$$

In Step $a$, we use $y_{i}=a_{i}+b_{i} \omega$ and Lemma 1.1 to obtain

$$
f\left(\left(y_{i}-\mathbf{x}_{i}^{T} \boldsymbol{\beta}\right) / \sigma\right)=f\left(\left(b_{i} \omega-\mathbf{x}_{i}^{T} \boldsymbol{\beta}\right) / \sigma+a_{i} / \sigma\right) \leq D\left(\left|a_{i}\right|, 1\right) f\left(\left(b_{i} \omega-\mathbf{x}_{i}^{T} \boldsymbol{\beta}\right) / \sigma\right),
$$

because $\left|a_{i} / \sigma\right| \leq\left|a_{i}\right|$ for all $i$. We also use $\pi(\boldsymbol{\beta}, \sigma) \leq B \max (1,1 / \sigma)=B$. In Step $b$, we use again Lemma 1.1 to obtain $f(\omega) / f\left(y_{i}\right)=f\left(\left(y_{i}-a_{i}\right) / b_{i}\right) / f\left(y_{i}\right) \leq\left|b_{i}\right| D\left(\left|a_{i}\right|,\left|b_{i}\right|\right)$. In Step $c$, we set $b_{i}=0$ if $k_{i}=1$ and we use the symmetry of $f$ to obtain $f\left(-\mathbf{x}_{i}^{T} \boldsymbol{\beta} / \sigma\right)=$ $f\left(\mathbf{x}_{i}^{T} \boldsymbol{\beta} / \sigma\right)$. In Step $d$, we use the assumption $k_{1}=\ldots=k_{p}=1$.

Now it suffices to demonstrate that

$$
\left[\frac{\omega / \sigma}{\omega f(\omega)}\right]^{\ell} \frac{1}{\sigma^{1 / 2}} \prod_{i=p+1}^{n}\left[f\left(\mathbf{x}_{i}^{T} \boldsymbol{\beta} / \sigma\right)\right]^{k_{i}}\left[f\left(\left(b_{i} \omega-\mathbf{x}_{i}^{T} \boldsymbol{\beta}\right) / \sigma\right)\right]^{\ell_{i}}
$$


is bounded by a constant that does not depend on $\omega, \boldsymbol{\beta}$ and $\sigma$ since $\prod_{i=1}^{p}(1 / \sigma) f\left(\mathbf{x}_{i}^{T} \boldsymbol{\beta} / \sigma\right)$ $\times(1 / \sigma)^{k-p-1 / 2}$ is an integrable function on area 1 . Indeed, since $k>p+1$, we have

$$
\begin{array}{r}
\int_{1}^{\infty}(1 / \sigma)^{k-p-1 / 2} \int_{\mathbb{R}^{p}} \prod_{i=1}^{p}(1 / \sigma) f\left(\mathbf{x}_{i}^{T} \boldsymbol{\beta} / \sigma\right) d \boldsymbol{\beta} d \sigma \\
=\left|\operatorname{det}\left(\begin{array}{c}
\mathbf{x}_{1}^{T} \\
\vdots \\
\mathbf{x}_{p}^{T}
\end{array}\right)\right|^{-1} \int_{1}^{\infty} \frac{1}{\sigma^{k-p-1 / 2}} d \sigma<\infty
\end{array}
$$

using the following change of variables: $u_{i}=\mathbf{x}_{i}^{T} \boldsymbol{\beta} / \sigma, i=1, \ldots, p$. The determinant is different from 0 because all the explanatory variables are continuous. When any type of explanatory variables is considered, we assume without loss of generality that $\mathbf{x}_{1}, \ldots, \mathbf{x}_{p}$ are additionally linearly independent. Note that if instead, in Step $a$ above, we bound $\pi(\boldsymbol{\beta}, \sigma)$ by $B / \sigma$, one can verify that the condition $k \geq p+1$ is sufficient to bound above the integral.

In order to bound the function in (1.3), we split Area 1 into three parts: $1 \leq \sigma<\omega^{1 / 2}$, $\omega^{1 / 2} \leq \sigma<\omega /(\gamma M)$ and $\omega /(\gamma M) \leq \sigma<\infty$, where $M$ is defined in (1.1) and $\gamma$ is a positive constant that can be chosen as large as we want (lower bounds are provided in the proof). Note that this split is well defined if $\mathrm{y}>\max \left(1,(\gamma M)^{2}\right)$ because $\omega \geq \mathrm{y}$.

First, consider $\omega /(\gamma M) \leq \sigma<\infty$. We have

$$
\begin{gathered}
{\left[\frac{\omega / \sigma}{\omega f(\omega)}\right]^{\ell} \frac{1}{\sigma^{1 / 2}} \prod_{i=p+1}^{n}\left[f\left(\mathbf{x}_{i}^{T} \boldsymbol{\beta} / \sigma\right)\right]^{k_{i}}\left[f\left(\left(b_{i} \omega-\mathbf{x}_{i}^{T} \boldsymbol{\beta}\right) / \sigma\right)\right]^{\ell_{i}} \stackrel{a}{\leq} \frac{B^{n-p}}{\sigma^{1 / 2}}\left[\frac{\omega / \sigma}{\omega f(\omega)}\right]^{\ell}} \\
\quad \stackrel{b}{\leq} B^{n-p}(\gamma M)^{\ell+1 / 2} \frac{(1 / \omega)^{1 / 2}}{[\omega f(\omega)]^{\ell}} \stackrel{c}{\leq} B^{n-p}(\gamma M)^{\ell+1 / 2} \frac{(1 / \omega)^{1 / 2}}{(\log \omega)^{-(\rho+1) \ell}} \\
\stackrel{d}{\leq} B^{n-p}(\gamma M)^{\ell+1 / 2}[2(\rho+1) \ell / \mathrm{e}]^{(\rho+1) \ell}<\infty .
\end{gathered}
$$

In Step $a$, we use $f \leq B$. In Step $b$, we use $\omega / \sigma \leq \gamma M$ and $1 / \sigma \leq \gamma M / \omega$. In Step $c$, we use $\omega f(\omega)>(\log \omega)^{-\rho-1}$ if $\omega \geq \mathrm{y} \geq A(1)$, where $A(1)$ comes from Proposition 1.1. For Step $d$, it is purely algebraic to show that the maximum of $(\log \omega)^{\xi} / \omega^{1 / 2}$ is $(2 \xi / \mathrm{e})^{\xi}$ for $\omega>1$ and $\xi>0$, where $\xi=(\rho+1) \ell$ in our situation.

Now, consider the two other parts combined (we will split them in the next step), that is $1 \leq \sigma \leq \omega /(\gamma M)$. We have

$$
\begin{aligned}
& {\left[\frac{\omega / \sigma}{\omega f(\omega)}\right]^{\ell} \frac{1}{\sigma^{1 / 2}} \prod_{i=p+1}^{n}\left[f\left(\mathbf{x}_{i}^{T} \boldsymbol{\beta} / \sigma\right)\right]^{k_{i}}\left[f\left(\left(b_{i} \omega-\mathbf{x}_{i}^{T} \boldsymbol{\beta}\right) / \sigma\right)\right]^{\ell_{i}}} \\
& \quad=\frac{1}{\sigma^{1 / 2}}\left[\frac{(\omega / \sigma) f(\omega / \sigma)}{\omega f(\omega)}\right]^{\ell} \prod_{i=p+1}^{n}\left[f\left(\mathbf{x}_{i}^{T} \boldsymbol{\beta} / \sigma\right)\right]^{k_{i}}\left[\frac{f\left(\left(b_{i} \omega-\mathbf{x}_{i}^{T} \boldsymbol{\beta}\right) / \sigma\right)}{f(\omega / \sigma)}\right]^{\ell_{i}} \\
& \quad \stackrel{a}{\leq} \frac{1}{\sigma^{1 / 2}}\left[\frac{(\omega / \sigma) f(\omega / \sigma)}{\omega f(\omega)}\right]^{\ell} B^{k-p}[D(0, \gamma) \gamma]^{\ell} .
\end{aligned}
$$


In Step $a$, we use

$$
\prod_{i=p+1}^{n}\left[f\left(\mathbf{x}_{i}^{T} \boldsymbol{\beta} / \sigma\right)\right]^{k_{i}}\left[\frac{f\left(\left(b_{i} \omega-\mathbf{x}_{i}^{T} \boldsymbol{\beta}\right) / \sigma\right)}{f(\omega / \sigma)}\right]^{\ell_{i}} \leq B^{k-p}[D(0, \gamma) \gamma]^{\ell} .
$$

The proof of this inequality is substantial. Therefore, to ease the reading, it is deferred after the demonstration that the remaining term, i.e.

$$
\frac{1}{\sigma^{1 / 2}}\left[\frac{(\omega / \sigma) f(\omega / \sigma)}{\omega f(\omega)}\right]^{\ell}
$$

is bounded.

We first consider $\omega^{1 / 2} \leq \sigma \leq \omega /(\gamma M)$. We have

$$
\frac{1}{\sigma^{1 / 2}}\left[\frac{(\omega / \sigma) f(\omega / \sigma)}{\omega f(\omega)}\right]^{\ell} \stackrel{a}{\leq} B^{\ell} \frac{(1 / \omega)^{1 / 4}}{[\omega f(\omega)]^{\ell}} \stackrel{b}{\leq} B^{\ell} \frac{(1 / \omega)^{1 / 4}}{(\log \omega)^{-(\rho+1) \ell}} \stackrel{c}{\leq} B^{\ell}[4(\rho+1) \ell / \mathrm{e}]^{(\rho+1) \ell}<\infty .
$$

In Step $a$, we use $(\omega / \sigma) f(\omega / \sigma) \leq B$ and $(1 / \sigma)^{1 / 2} \leq(1 / \omega)^{1 / 4}$. In Step $b$, we use $\omega f(\omega)>$ $(\log \omega)^{-\rho-1}$ if $\omega \geq \mathrm{y} \geq A(1)$, where $A(1)$ comes from Proposition 1.1. In Step $c$, it is purely algebraic to show that the maximum of $(\log \omega)^{\xi} / \omega^{1 / 4}$ is $(4 \xi / \mathrm{e})^{\xi}$ for $\omega>1$ and $\xi>0$, where $\xi=(\rho+1) \ell$ in our situation.

We now consider $1 \leq \sigma \leq \omega^{1 / 2}$. We have

$$
\frac{1}{\sigma^{1 / 2}}\left[\frac{(\omega / \sigma) f(\omega / \sigma)}{\omega f(\omega)}\right]^{\ell} \stackrel{a}{\leq}\left[\frac{\omega^{1 / 2} f\left(\omega^{1 / 2}\right)}{\omega f(\omega)}\right]^{\ell} \stackrel{b}{\leq} 2^{(\rho+1) \ell}<\infty .
$$

In Step $a$, we use $1 / \sigma \leq 1$ and $(\omega / \sigma) f(\omega / \sigma) \leq \omega^{1 / 2} f\left(\omega^{1 / 2}\right)$ by the monotonicity of the tails of $|z| f(z)$ since $\omega / \sigma \geq \omega^{1 / 2} \geq \mathrm{y}^{1 / 2} \geq M$ if $\mathrm{y} \geq M^{2}$. In Step $b$, we use $\omega^{1 / 2} f\left(\omega^{1 / 2}\right) /(\omega f(\omega)) \leq 2(1 / 2)^{-\rho}=2^{\rho+1}$ if $\omega \geq \mathrm{y} \geq A$, where $A$ is a positive constant, see the definition of log-regularly varying functions (Definition 2.1 in our paper).

Finally, we prove the inequality in (1.4). Recall that we assumed without loss of generality that the first $\ell+2 p-1$ observations are nonoutliers (therefore $k_{1}=\ldots=$ $k_{\ell+2 p-1}=1$ ). We know that $\mathbf{x}_{1}, \ldots, \mathbf{x}_{p}$ have been used earlier to integrate over $\boldsymbol{\beta}$ and $\sigma$. We also know that there are at least $p$ remaining nonoutliers among observations 1 to $\ell+2 p-1$ because $\ell+2 p-1-p=\ell+p-1 \geq p$ (because we assume that $\ell \geq 1$ ).

In order to prove the result, we split the domain of $\boldsymbol{\beta}$ as follows:

$$
\begin{gathered}
\mathbb{R}^{p}=\left[\cap_{i} \mathcal{O}_{i}^{c}\right] \cup\left[\cup_{i}\left(\mathcal{O}_{i} \cap\left(\cap_{i_{1}} \mathcal{F}_{i_{1}}^{c}\right)\right)\right] \cup\left[\cup_{i, i_{1}}\left(\mathcal{O}_{i} \cap \mathcal{F}_{i_{1}} \cap\left(\cap_{i_{2} \neq i_{1}} \mathcal{F}_{i_{2}}^{c}\right)\right)\right] \\
\cup \cdots \cup\left[\cup_{i, i_{1}, \ldots, i_{p-1}\left(i_{j} \neq i_{s} \forall i_{j}, i_{s} \text { s.t. } j \neq s\right)}\left(\mathcal{O}_{i} \cap \mathcal{F}_{i_{1}} \cap \cdots \cap \mathcal{F}_{i_{p-1}} \cap\left(\cap_{i_{p} \neq i_{1}, \ldots, i_{p-1}} \mathcal{F}_{i_{p}}^{c}\right)\right)\right] \\
\cup\left[\cup_{i, i_{1}, \ldots, i_{p}\left(i_{j} \neq i_{s} \forall i_{j}, i_{s} \text { s.t. } j \neq s\right)}\left(\mathcal{O}_{i} \cap \mathcal{F}_{i_{1}} \cap \cdots \cap \mathcal{F}_{i_{p}}\right)\right],
\end{gathered}
$$

where

$$
\mathcal{O}_{i}:=\left\{\boldsymbol{\beta}:\left|b_{i} \omega-\mathbf{x}_{i}^{T} \boldsymbol{\beta}\right|<\omega / 2\right\}, \forall i \in \mathcal{I}_{\mathcal{O}},
$$




$$
\mathcal{F}_{i}:=\left\{\boldsymbol{\beta}:\left|\mathbf{x}_{i}^{T} \boldsymbol{\beta}\right|<\omega / \gamma\right\}, \forall i \in \mathcal{I}_{\mathcal{F}},
$$

$\mathcal{I}_{\mathcal{O}}:=\left\{i: i \in\{\ell+2 p-1, \ldots, n\}\right.$ and $\left.\ell_{i}=1\right\}$ and $\mathcal{I}_{\mathcal{F}}:=\{p+1, \ldots, \ell+2 p-1\}$ are the sets of indexes of outliers and remaining fixed observations (nonoutliers) among observations 1 to $\ell+2 p-1$, respectively.

The set $\mathcal{O}_{i}$ represents the hyperplanes $y=\mathbf{x}_{i}^{T} \boldsymbol{\beta}$ characterised by the different values of $\boldsymbol{\beta}$ that satisfy $\left|b_{i} \omega-\mathbf{x}_{i}^{T} \boldsymbol{\beta}\right|<\omega / 2$. In other words, it represents the hyperplanes that pass at a vertical distance of less than $\omega / 2$ of the point $\left(\mathbf{x}_{i}, b_{i} \omega\right)$, which is considered as an outlier since $\omega \rightarrow \infty$ (recall that $b_{i} \omega=y_{i}-a_{i}$ ). Analogously, the set $\mathcal{F}_{i}$ represents the hyperplanes that pass at a vertical distance of less than $\omega / \gamma$ of the point $\left(\mathbf{x}_{i}, 0\right)$, which is considered as a nonoutlier. Therefore, the set $\cap_{i} \mathcal{O}_{i}^{c}$ represents the hyperplanes that pass at a vertical distance of at least $\omega / 2$ of all the points $\left(\mathbf{x}_{i}, b_{i} \omega\right)$ (all the outliers). The set $\cup_{i}\left(\mathcal{O}_{i} \cap\left(\cap_{i_{1}} \mathcal{F}_{i_{1}}^{c}\right)\right)$ represents the hyperplanes that pass at a vertical distance of less than $\omega / 2$ of at least one point $\left(\mathbf{x}_{i}, b_{i} \omega\right)$ (an outlier), but at a vertical distance of at least $\omega / \gamma$ of all the points $\left(\mathbf{x}_{i}, 0\right)$ (all the nonoutliers). For each $i_{1} \in \mathcal{I}_{\mathcal{F}}$, the set $\cup_{i}\left(\mathcal{O}_{i} \cap \mathcal{F}_{i_{1}} \cap\left(\cap_{i_{2} \neq i_{1}} \mathcal{F}_{i_{2}}^{c}\right)\right)$ represents the hyperplanes that pass at a vertical distance of less than $\omega / 2$ of at least one point $\left(\mathbf{x}_{i}, b_{i} \omega\right)$ (an outlier), at a vertical distance of less than $\omega / \gamma$ of the point $\left(\mathbf{x}_{i_{1}}, 0\right)$ (a nonoutlier), but at a vertical distance of at least $\omega / \gamma$ of all the other nonoutliers, and so on.

Now, we claim that $\mathcal{O}_{i} \cap \mathcal{F}_{i_{1}} \cap \cdots \cap \mathcal{F}_{i_{p}}=\varnothing$ for all $i, i_{1}, \ldots, i_{p}$ with $i_{j} \neq i_{s}, \forall i_{j}, i_{s}$ such that $j \neq s$, meaning that there is no hyperplane that passes at a vertical distance of less than $\omega / 2$ of the point $\left(\mathbf{x}_{i}, b_{i} \omega\right)$ (an outlier) and at the same at a vertical distance of less than $\omega / \gamma$ of $p$ points $\left(\mathbf{x}_{i_{j}}, 0\right)$ (nonoutliers). To prove this, we use the fact that $\mathbf{x}_{i}$ (a vector of size $p$ ) can be expressed as a linear combination of $\mathbf{x}_{i_{1}}, \ldots, \mathbf{x}_{i_{p}}$. This is true because all explanatory variables are continuous, and therefore, linearly independent with probability 1 . When any type of explanatory variables is considered, we select observations 1 to $\ell+2 p-1$ to be such that any $p$ vectors $\mathbf{x}_{i_{1}}, \ldots, \mathbf{x}_{i_{p}}$, with $\left\{i_{1}, \ldots, i_{p}\right\} \subset$ $\{1, \ldots, \ell+2 p-1\}$, are linearly independent. This is possible given the assumption mentioned in Remark 2.2. As a result, considering that $\boldsymbol{\beta} \in \mathcal{F}_{i_{1}} \cap \cdots \cap \mathcal{F}_{i_{p}}$ and $\mathbf{x}_{i}=$ $\sum_{s=1}^{p} a_{s} \mathbf{x}_{i_{s}}$ for some $a_{1}, \ldots, a_{p} \in \mathbb{R}$, we have

$$
\begin{gathered}
\left|b_{i} \omega-\mathbf{x}_{i}^{T} \boldsymbol{\beta}\right|=\left|b_{i} \omega-\left(\sum_{s=1}^{p} a_{s} \mathbf{x}_{i_{s}}\right)^{T} \boldsymbol{\beta}\right| \stackrel{a}{\geq}\left|b_{i} \omega\right|-\left|\sum_{s=1}^{p} a_{s} \mathbf{x}_{i_{s}}^{T} \boldsymbol{\beta}\right| \stackrel{b}{\geq} \omega-\frac{\omega}{\gamma} \sum_{s=1}^{p}\left|a_{s}\right| \\
\geq \omega-\frac{\omega}{2} .
\end{gathered}
$$

In Step $a$, we use the reverse triangle inequality. In Step $b$, we use that $\left|b_{i}\right| \geq 1$ and $\left|\sum_{s=1}^{p} a_{s} \mathbf{x}_{i_{s}}^{T} \boldsymbol{\beta}\right| \leq \sum_{s=1}^{p}\left|a_{s}\right|\left|\mathbf{x}_{i_{s}}^{T} \boldsymbol{\beta}\right| \leq \sum_{s=1}^{p}\left|a_{s}\right| \omega / \gamma$ because $\boldsymbol{\beta} \in \mathcal{F}_{i_{1}} \cap \cdots \cap \mathcal{F}_{i_{p}}$, which means that $\left|\mathbf{x}_{i}^{T} \boldsymbol{\beta}\right|<\omega / \gamma$ for all $i \in\left\{i_{1}, \ldots, i_{p}\right\}$. In Step $c$, we define the constant $\gamma$ such that $\gamma \geq 2 \sum_{s=1}^{p}\left|a_{s}\right|$ (we choose $\gamma$ such that it satisfies this inequality for any combination of $i$ and $\left.i_{1}, \ldots, i_{p}\right)$. Therefore, we have that $\boldsymbol{\beta} \notin \mathcal{O}_{i}$. This proves that $\mathcal{O}_{i} \cap \mathcal{F}_{i_{1}} \cap \cdots \cap \mathcal{F}_{i_{p}}=\varnothing$ for all $i, i_{1}, \ldots, i_{p}$ with $i_{j} \neq i_{s}, \forall i_{j}, i_{s}$ such that $j \neq s$. This in turn implies that (1.5) can be rewritten as

$$
\mathbb{R}^{p}=\left[\cap_{i} \mathcal{O}_{i}^{c}\right] \cup\left[\cup_{i}\left(\mathcal{O}_{i} \cap\left(\cap_{i_{1}} \mathcal{F}_{i_{1}}^{c}\right)\right)\right] \cup\left[\cup_{i, i_{1}}\left(\mathcal{O}_{i} \cap \mathcal{F}_{i_{1}} \cap\left(\cap_{i_{2} \neq i_{1}} \mathcal{F}_{i_{2}}^{c}\right)\right)\right]
$$




$$
\cup \cdots \cup\left[\cup_{i, i_{1}, \ldots, i_{p-1}\left(i_{j} \neq i_{s} \forall i_{j}, i_{s} \text { s.t. } j \neq s\right)}\left(\mathcal{O}_{i} \cap \mathcal{F}_{i_{1}} \cap \cdots \cap \mathcal{F}_{i_{p-1}} \cap\left(\cap_{i_{p} \neq i_{1}, \ldots, i_{p-1}} \mathcal{F}_{i_{p}}^{c}\right)\right)\right] .
$$

This decomposition of $\mathbb{R}^{p}$ is comprised of $1+\sum_{i=0}^{p-1}\left({ }^{\ell+p-1}{ }_{i}\right)$ mutually exclusive sets given by $\cap_{i} \mathcal{O}_{i}^{c}, \cup_{i}\left(\mathcal{O}_{i} \cap\left(\cap_{i_{1}} \mathcal{F}_{i_{1}}^{c}\right)\right), \cup_{i}\left(\mathcal{O}_{i} \cap \mathcal{F}_{i_{1}} \cap\left(\cap_{i_{2} \neq i_{1}} \mathcal{F}_{i_{2}}^{c}\right)\right)$ for $i_{1} \in \mathcal{I}_{\mathcal{F}}$, and so on.

We are now ready to bound the function on the left-hand side in (1.4). We first show that the function is bounded on $\boldsymbol{\beta} \in \cap_{i} \mathcal{O}_{i}^{c}$ and $1 \leq \sigma \leq \omega /(\gamma M)$. For all $i \in \mathcal{I}_{\mathcal{O}}$, we have

$$
\frac{f\left(\left(b_{i} \omega-\mathbf{x}_{i}^{T} \boldsymbol{\beta}\right) / \sigma\right)}{f(\omega / \sigma)} \leq \frac{f(\omega /(2 \sigma))}{f(\omega / \sigma)} \leq 2 D(0,2) \leq D(0, \gamma) \gamma
$$

using the monotonicity of $f$ because $\left|b_{i} \omega-\mathbf{x}_{i}^{T} \boldsymbol{\beta}\right| / \sigma \geq \omega /(2 \sigma) \geq \gamma M / 2 \geq M$ (we choose $\gamma \geq 2)$, and then Lemma 1.1. Therefore, on $\boldsymbol{\beta} \in \cap_{i} \mathcal{O}_{i}^{c}$ and $1 \leq \sigma \leq \omega /(\gamma M)$,

$$
\prod_{i=p+1}^{n}\left[f\left(\mathbf{x}_{i}^{T} \boldsymbol{\beta} / \sigma\right)\right]^{k_{i}}\left[\frac{f\left(\left(b_{i} \omega-\mathbf{x}_{i}^{T} \boldsymbol{\beta}\right) / \sigma\right)}{f(\omega / \sigma)}\right]^{\ell_{i}} \leq B^{k-p}[D(0, \gamma) \gamma]^{\ell},
$$

using $f \leq B$.

Now, we consider the area defined by: $1 \leq \sigma \leq \omega /(\gamma M)$ and $\boldsymbol{\beta}$ belongs to one of the $\sum_{i=0}^{p-1}\left(\begin{array}{c}k-p \\ i\end{array}\right)$ mutually exclusive sets $\cup_{i}\left(\mathcal{O}_{i} \cap\left(\cap_{i_{1}} \mathcal{F}_{i_{1}}^{c}\right)\right), \cup_{i}\left(\mathcal{O}_{i} \cap \mathcal{F}_{i_{1}} \cap\left(\cap_{i_{2} \neq i_{1}} \mathcal{F}_{i_{2}}^{c}\right)\right)$ for $i_{1} \in \mathcal{I}_{\mathcal{F}}$, etc. We have

$$
\begin{aligned}
\prod_{i=p+1}^{n}\left[f\left(\mathbf{x}_{i}^{T} \boldsymbol{\beta} / \sigma\right)\right]^{k_{i}}\left[\frac{f\left(\left(b_{i} \omega-\mathbf{x}_{i}^{T} \boldsymbol{\beta}\right) / \sigma\right)}{f(\omega / \sigma)}\right]^{\ell_{i}} & \stackrel{a}{\leq} B^{\ell} \prod_{i=p+1}^{n} \frac{\left[f\left(\mathbf{x}_{i}^{T} \boldsymbol{\beta} / \sigma\right)\right]^{k_{i}}}{[f(\omega / \sigma)]^{\ell_{i}}} \\
& \stackrel{b}{\leq} B^{\ell+(k-p)-\ell}[D(0, \gamma) \gamma]^{\ell}=B^{k-p}[D(0, \gamma) \gamma]^{\ell} .
\end{aligned}
$$

In Step $a$, we use $f \leq B$ for all $i \in \mathcal{I}_{\mathcal{O}}$. In Step $b$, we use the fact that in any of the sets in which $\boldsymbol{\beta}$ can belong, there are at least $\ell$ nonoutlying points $\left(\mathbf{x}_{i}, 0\right)$ such that $\left|\mathbf{x}_{i}^{T} \boldsymbol{\beta}\right| \geq \omega / \gamma$. Indeed, the case in which there are the least nonoutliers such that $\left|\mathbf{x}_{i}^{T} \boldsymbol{\beta}\right| \geq \omega / \gamma$ corresponds to $\boldsymbol{\beta} \in \cup_{i}\left(\mathcal{O}_{i} \cap \mathcal{F}_{i_{1}} \cap \cdots \cap \mathcal{F}_{i_{p-1}} \cap\left(\cap_{i_{p} \neq i_{1}, \ldots, i_{p-1}} \mathcal{F}_{i_{p}}^{c}\right)\right)$. In this case there are $p-1$ nonoutliers such that $\left|\mathbf{x}_{i}^{T} \boldsymbol{\beta}\right|<\omega / \gamma$ (observations $i_{1}$ to $i_{p-1}$ ), which leaves $\ell+p-1-(p-1)=\ell$ nonoutliers such that $\left|\mathbf{x}_{i}^{T} \boldsymbol{\beta}\right| \geq \omega / \gamma$ (i.e. that there are $\ell$ sets in the intersection $\cap_{i_{p} \neq i_{1}, \ldots, i_{p-1}} \mathcal{F}_{i_{p}}^{c}$ ). Therefore, for $\ell$ nonoutliers such that $\left|\mathbf{x}_{i}^{T} \boldsymbol{\beta}\right| \geq \omega / \gamma$, we use

$$
f\left(\mathbf{x}_{i}^{T} \boldsymbol{\beta} / \sigma\right) / f(\omega / \sigma) \leq f(\omega /(\gamma \sigma)) / f(\omega / \sigma) \leq D(0, \gamma) \gamma,
$$

by the monotonicity of $f$ because $\left|\mathbf{x}_{i}^{T} \boldsymbol{\beta}\right| / \sigma \geq \omega /(\gamma \sigma) \geq M$, and then Lemma 1.1. For the remaining $k-p-\ell$ nonoutlying points, we use $f \leq B$. Note that this argument justifies the need of the assumption $k \geq \ell+2 p-1$.

Area 2: Consider $0<\sigma<1$. We actually need to show that

$$
\lim _{\omega \rightarrow \infty} \int_{\mathbb{R}^{p}} \int_{0}^{1} \pi\left(\boldsymbol{\beta}, \sigma \mid \mathbf{y}_{\mathbf{k}}\right) \prod_{i=1}^{n}\left[\frac{(1 / \sigma) f\left(\left(y_{i}-\mathbf{x}_{i}^{T} \boldsymbol{\beta}\right) / \sigma\right)}{f\left(y_{i}\right)}\right]^{\ell_{i}} d \sigma d \boldsymbol{\beta}
$$




$$
=\int_{\mathbb{R}^{p}} \int_{0}^{1} \pi\left(\boldsymbol{\beta}, \sigma \mid \mathbf{y}_{\mathbf{k}}\right) d \sigma d \boldsymbol{\beta} .
$$

For Area 2, we proceed in a slightly different manner than for Area 1. We begin by dividing the first integral above into two parts as follows:

$$
\begin{aligned}
\lim _{\omega \rightarrow \infty} & \int_{\mathbb{R}^{p}} \int_{0}^{1} \pi\left(\boldsymbol{\beta}, \sigma \mid \mathbf{y}_{\mathbf{k}}\right) \prod_{i=1}^{n}\left[\frac{(1 / \sigma) f\left(\left(y_{i}-\mathbf{x}_{i}^{T} \boldsymbol{\beta}\right) / \sigma\right)}{f\left(y_{i}\right)}\right]^{\ell_{i}} d \sigma d \boldsymbol{\beta} \\
= & \lim _{\omega \rightarrow \infty} \int_{\mathbb{R}^{p}} \int_{0}^{1} \pi\left(\boldsymbol{\beta}, \sigma \mid \mathbf{y}_{\mathbf{k}}\right) \prod_{i=1}^{n}\left[\frac{(1 / \sigma) f\left(\left(y_{i}-\mathbf{x}_{i}^{T} \boldsymbol{\beta}\right) / \sigma\right)}{f\left(y_{i}\right)}\right]^{\ell_{i}} \mathbb{1}_{\cap_{i} \mathcal{O}_{i}^{c}}(\boldsymbol{\beta}) d \sigma d \boldsymbol{\beta} \\
& +\lim _{\omega \rightarrow \infty} \int_{\cup_{i} \mathcal{O}_{i}} \int_{0}^{1} \pi\left(\boldsymbol{\beta}, \sigma \mid \mathbf{y}_{\mathbf{k}}\right) \prod_{i=1}^{n}\left[\frac{(1 / \sigma) f\left(\left(y_{i}-\mathbf{x}_{i}^{T} \boldsymbol{\beta}\right) / \sigma\right)}{f\left(y_{i}\right)}\right]^{\ell_{i}} d \sigma d \boldsymbol{\beta},
\end{aligned}
$$

where

$$
\mathcal{O}_{i}:=\left\{\boldsymbol{\beta}:\left|y_{i}-\mathbf{x}_{i}^{T} \boldsymbol{\beta}\right|<\omega / 2\right\}, \forall i \in \mathcal{I}_{\mathcal{O}},
$$

with $\mathcal{I}_{\mathcal{O}}:=\left\{i: i \in\{1, \ldots, n\}\right.$ and $\left.\ell_{i}=1\right\}$. Note that the definition of $\mathcal{O}_{i}$ is very similar as that of the set defined in (1.6) (this is why we use the same notation); its interpretation is also very similar. We show that the first part above is equal to the integral $\int_{\mathbb{R}^{p}} \int_{0}^{1} \pi\left(\boldsymbol{\beta}, \sigma \mid \mathbf{y}_{\mathbf{k}}\right) d \sigma d \boldsymbol{\beta}$ and that the second part is equal to 0 .

For the first part, we again use Lebesgue's dominated convergence theorem in order to interchange the limit $\omega \rightarrow \infty$ and the integral. We have

$$
\begin{gathered}
\lim _{\omega \rightarrow \infty} \int_{\mathbb{R}^{p}} \int_{0}^{1} \pi\left(\boldsymbol{\beta}, \sigma \mid \mathbf{y}_{\mathbf{k}}\right) \prod_{i=1}^{n}\left[\frac{(1 / \sigma) f\left(\left(y_{i}-\mathbf{x}_{i}^{T} \boldsymbol{\beta}\right) / \sigma\right)}{f\left(y_{i}\right)}\right]^{\ell_{i}} \mathbb{1}_{\cap_{i} \mathcal{O}_{i}^{c}}(\boldsymbol{\beta}) d \sigma d \boldsymbol{\beta} \\
=\int_{\mathbb{R}^{p}} \int_{0}^{1} \pi\left(\boldsymbol{\beta}, \sigma \mid \mathbf{y}_{\mathbf{k}}\right) \lim _{\omega \rightarrow \infty} \prod_{i=1}^{n}\left[\frac{(1 / \sigma) f\left(\left(y_{i}-\mathbf{x}_{i}^{T} \boldsymbol{\beta}\right) / \sigma\right)}{f\left(y_{i}\right)}\right]^{\ell_{i}} \mathbb{1}_{\cap_{i} \mathcal{O}_{i}^{c}}(\boldsymbol{\beta}) d \sigma d \boldsymbol{\beta} \\
=\int_{\mathbb{R}^{p}} \int_{0}^{1} \pi\left(\boldsymbol{\beta}, \sigma \mid \mathbf{y}_{\mathbf{k}}\right) \times 1 \times \mathbb{1}_{\mathbb{R}^{p}}(\boldsymbol{\beta}) d \sigma d \boldsymbol{\beta}=\int_{\mathbb{R}^{p}} \int_{0}^{1} \pi\left(\boldsymbol{\beta}, \sigma \mid \mathbf{y}_{\mathbf{k}}\right) d \sigma d \boldsymbol{\beta},
\end{gathered}
$$

using Proposition 2.2 in our paper in the second equality since $\mathbf{x}_{1}, \ldots, \mathbf{x}_{n}$ are fixed, and $\lim _{\omega \rightarrow \infty} \mathbb{1}_{\cap_{i} \mathcal{O}_{i}^{c}}(\boldsymbol{\beta})=\mathbb{1}_{\mathbb{R}^{p}}(\boldsymbol{\beta})=1 \Leftrightarrow \lim _{\omega \rightarrow \infty} \mathbb{1}_{\cup_{i} \mathcal{O}_{i}}(\boldsymbol{\beta})=0$. Indeed, if $\ell_{i}=1$ and $b_{i}>0$ (which implies that $y_{i}>0$ ), $\boldsymbol{\beta} \in \mathcal{O}_{i}$ implies that $\left|y_{i}-\mathbf{x}_{i}^{T} \boldsymbol{\beta}\right|<\omega / 2 \leq y_{i} / 2$, which in turn implies that $y_{i} / 2<\mathbf{x}_{i}^{T} \boldsymbol{\beta}<3 y_{i} / 2$, and in the limit, no $\boldsymbol{\beta} \in \mathbb{R}^{p}$ satisfies this (we have the same conclusion if $b_{i}<0$ ). Note that pointwise convergence is sufficient, for any value of $\boldsymbol{\beta} \in \mathbb{R}^{p}$ and $\sigma>0$, once the limit is inside the integral. We now demonstrate that the integrand is bounded, for any value of $\omega \geq \mathrm{y}$, by an integrable function of $\boldsymbol{\beta}$ and $\sigma$ that does not depend on $\omega$.

Consider $\boldsymbol{\beta} \in \cap_{i} \mathcal{O}_{i}^{c}$, that is $\left\{\boldsymbol{\beta}:\left|y_{i}-\mathbf{x}_{i}^{T} \boldsymbol{\beta}\right| \geq \omega / 2\right.$ for all $\left.i \in \mathcal{I}_{\mathcal{O}}\right\}$, and $0<\sigma<1$. Note that the integrand is equal to 0 if $\boldsymbol{\beta} \notin \cap_{i} \mathcal{O}_{i}^{c}$. For all $i \in \mathcal{I}_{\mathcal{O}}$, we have

$$
(1 / \sigma) f\left(\left(y_{i}-\mathbf{x}_{i}^{T} \boldsymbol{\beta}\right) / \sigma\right) \leq f\left(y_{i}-\mathbf{x}_{i}^{T} \boldsymbol{\beta}\right) \leq f(\omega / 2) \leq 2\left|b_{i}\right| D\left(\left|a_{i}\right|, 2\left|b_{i}\right|\right) f\left(y_{i}\right),
$$


by the monotonicity of the tails of $|z| f(z)$ and then the monotonicity of the tails of $f(z)$, because $\left|y_{i}-\mathbf{x}_{i}^{T} \boldsymbol{\beta}\right| / \sigma \geq\left|y_{i}-\mathbf{x}_{i}^{T} \boldsymbol{\beta}\right| \geq \omega / 2 \geq \mathrm{y} / 2 \geq M$, if we choose $\mathrm{y} \geq 2 M$. Lemma 1.1 is used in the last inequality with $\omega=\left(y_{i}-a_{i}\right) / b_{i}$. Therefore,

$$
\begin{gathered}
\pi\left(\boldsymbol{\beta}, \sigma \mid \mathbf{y}_{\mathbf{k}}\right) \prod_{i=1}^{n}\left[\frac{(1 / \sigma) f\left(\left(y_{i}-\mathbf{x}_{i}^{T} \boldsymbol{\beta}\right) / \sigma\right)}{f\left(y_{i}\right)}\right]^{\ell_{i}} \mathbb{1}_{\cap_{i} \mathcal{O}_{i}^{c}}(\boldsymbol{\beta}) \\
\leq \pi\left(\boldsymbol{\beta}, \sigma \mid \mathbf{y}_{\mathbf{k}}\right) \prod_{i=1}^{n}\left[2\left|b_{i}\right| D\left(\left|a_{i}\right|, 2\left|b_{i}\right|\right)\right]^{\ell_{i}},
\end{gathered}
$$

which is an integrable function.

We now prove that

$$
\lim _{\omega \rightarrow \infty} \int_{\cup_{i} \mathcal{O}_{i}} \int_{0}^{1} \pi\left(\boldsymbol{\beta}, \sigma \mid \mathbf{y}_{\mathbf{k}}\right) \prod_{i=1}^{n}\left[\frac{(1 / \sigma) f\left(\left(y_{i}-\mathbf{x}_{i}^{T} \boldsymbol{\beta}\right) / \sigma\right)}{f\left(y_{i}\right)}\right]^{\ell_{i}} d \sigma d \boldsymbol{\beta}=0 .
$$

We first bound above the integrand and then we prove that the integral of the upper bound converges towards 0 as $\omega \rightarrow \infty$. In the same manner as in the proof of the inequality in (1.4), we split the domain of $\boldsymbol{\beta}$ as follows:

$$
\begin{gathered}
\cup_{i} \mathcal{O}_{i}=\left[\cup_{i}\left(\mathcal{O}_{i} \cap\left(\cap_{i_{1}} \mathcal{F}_{i_{1}}^{c}\right)\right)\right] \cup\left[\cup_{i, i_{1}}\left(\mathcal{O}_{i} \cap \mathcal{F}_{i_{1}} \cap\left(\cap_{i_{2} \neq i_{1}} \mathcal{F}_{i_{2}}^{c}\right)\right)\right] \\
\cup \cdots \cup\left[\cup_{i, i_{1}, \ldots, i_{p-1}\left(i_{j} \neq i_{s} \forall i_{j}, i_{s} \text { s.t. } j \neq s\right)}\left(\mathcal{O}_{i} \cap \mathcal{F}_{i_{1}} \cap \cdots \cap \mathcal{F}_{i_{p-1}} \cap\left(\cap_{i_{p} \neq i_{1}, \ldots, i_{p-1}} \mathcal{F}_{i_{p}}^{c}\right)\right)\right] \\
\cup\left[\cup_{i, i_{1}, \ldots, i_{p}\left(i_{j} \neq i_{s} \forall i_{j}, i_{s} \text { s.t. } j \neq s\right)}\left(\mathcal{O}_{i} \cap \mathcal{F}_{i_{1}} \cap \cdots \cap \mathcal{F}_{i_{p}}\right)\right],
\end{gathered}
$$

where

$$
\mathcal{F}_{i}:=\left\{\boldsymbol{\beta}:\left|\mathbf{x}_{i}^{T} \boldsymbol{\beta}\right|<\omega / \gamma\right\}, \forall i \in \mathcal{I}_{\mathcal{F}},
$$

and $\mathcal{I}_{\mathcal{F}}:=\{1, \ldots, \ell+2 p-1\}$ (we assume as previously that $y_{1}, \ldots, y_{\ell+2 p-1}$ are $\ell+2 p-1$ nonoutliers, and therefore $k_{1}=\ldots=k_{\ell+2 p-1}=1$ ). The definition of $\mathcal{F}_{i}$ is the same as that of the set defined in (1.7). For an interpretation of this set and of the sets involve in the decomposition of $\cup_{i} \mathcal{O}_{i}$, see the proof of (1.4). Given that $\left|y_{i}\right| \geq \omega$ for all $i \in \mathcal{O}_{i}$, we can use the same mathematical arguments as in the proof of (1.4) to show that $\mathcal{O}_{i} \cap \mathcal{F}_{i_{1}} \cap \cdots \cap \mathcal{F}_{i_{p}}=\varnothing$ for all $i, i_{1}, \ldots, i_{p}$ with $i_{j} \neq i_{s}, \forall i_{j} \neq i_{s}$ such that $j \neq s$. Therefore,

$$
\begin{aligned}
& \cup_{i} \mathcal{O}_{i}=\left[\cup_{i}\left(\mathcal{O}_{i} \cap\left(\cap_{i_{1}} \mathcal{F}_{i_{1}}^{c}\right)\right)\right] \cup\left[\cup_{i, i_{1}}\left(\mathcal{O}_{i} \cap \mathcal{F}_{i_{1}} \cap\left(\cap_{i_{2} \neq i_{1}} \mathcal{F}_{i_{2}}^{c}\right)\right)\right] \\
& \cup \cdots \cup\left[\cup_{i, i_{1}, \ldots, i_{p-1}\left(i_{j} \neq i_{s} \forall i_{j}, i_{s} \text { s.t. } j \neq s\right)}\left(\mathcal{O}_{i} \cap \mathcal{F}_{i_{1}} \cap \cdots \cap \mathcal{F}_{i_{p-1}} \cap\left(\cap_{i_{p} \neq i_{1}, \ldots, i_{p-1}} \mathcal{F}_{i_{p}}^{c}\right)\right)\right] .
\end{aligned}
$$

This decomposition of $\cup_{i} \mathcal{O}_{i}$ is comprised of $\sum_{i=0}^{p-1}\left({ }^{\ell+2 p-1}{ }_{i}\right)$ mutually exclusive sets given by $\cup_{i}\left(\mathcal{O}_{i} \cap\left(\cap_{i_{1}} \mathcal{F}_{i_{1}}^{c}\right)\right), \cup_{i}\left(\mathcal{O}_{i} \cap \mathcal{F}_{i_{1}} \cap\left(\cap_{i_{2} \neq i_{1}} \mathcal{F}_{i_{2}}^{c}\right)\right)$ for $i_{1} \in \mathcal{I}_{\mathcal{F}}$, and so on. We now consider the area defined by: $0<\sigma<1$ and $\boldsymbol{\beta}$ belongs to one of these $\sum_{i=0}^{p-1}\left(\begin{array}{c}\ell+2 p-1 \\ i\end{array}\right)$ mutually exclusive sets. We have

$$
\pi\left(\boldsymbol{\beta}, \sigma \mid \mathbf{y}_{\mathbf{k}}\right) \prod_{i=1}^{n}\left[\frac{(1 / \sigma) f\left(\left(y_{i}-\mathbf{x}_{i}^{T} \boldsymbol{\beta}\right) / \sigma\right)}{f\left(y_{i}\right)}\right]^{\ell_{i}}
$$




$$
\begin{aligned}
& \stackrel{a}{\leq} \pi\left(\boldsymbol{\beta}, \sigma \mid \mathbf{y}_{\mathbf{k}}\right) \prod_{i=1}^{n}\left[\frac{\left|b_{i}\right| D\left(\left|a_{i}\right|,\left|b_{i}\right|\right)(1 / \sigma) f\left(\left(y_{i}-\mathbf{x}_{i}^{T} \boldsymbol{\beta}\right) / \sigma\right)}{f(\omega)}\right]^{\ell_{i}} \\
& \propto \pi(\boldsymbol{\beta}, \sigma) \prod_{i=1}^{n}\left[(1 / \sigma) f\left(\left(a_{i}-\mathbf{x}_{i}^{T} \boldsymbol{\beta}\right) / \sigma\right)\right]^{k_{i}}\left[\frac{(1 / \sigma) f\left(\left(y_{i}-\mathbf{x}_{i}^{T} \boldsymbol{\beta}\right) / \sigma\right)}{f(\omega)}\right]^{\ell_{i}} \\
& \stackrel{n}{\leq}(B / \sigma)[2 \gamma D(0,2 \gamma)(1 / \sigma) f(\omega / \sigma)]^{\ell+1} \prod_{i=1\left(i \neq i_{p}, \ldots, i_{\ell+p}\right)}^{n}\left[(1 / \sigma) f\left(\left(a_{i}-\mathbf{x}_{i}^{T} \boldsymbol{\beta}\right) / \sigma\right)\right]^{k_{i}} \\
& \times\left[\frac{(1 / \sigma) f\left(\left(y_{i}-\mathbf{x}_{i}^{T} \boldsymbol{\beta}\right) / \sigma\right)}{f(\omega)}\right]^{\ell_{i}} \\
& \propto(1 / \sigma)[(1 / \sigma) f(\omega / \sigma)]^{\ell+1} \prod_{i=1\left(i \neq i_{p}, \ldots, i_{\ell+p}\right)}^{n}\left[(1 / \sigma) f\left(\left(a_{i}-\mathbf{x}_{i}^{T} \boldsymbol{\beta}\right) / \sigma\right)\right]^{k_{i}} \\
& \times\left[\frac{(1 / \sigma) f\left(\left(y_{i}-\mathbf{x}_{i}^{T} \boldsymbol{\beta}\right) / \sigma\right)}{f(\omega)}\right]^{\ell_{i}} \\
& \stackrel{c}{\leq}(1 / \sigma)(1 / \sigma) f(\omega / \sigma) \prod_{i=1\left(i \neq i_{p}, \ldots, i_{\ell+p}\right)}^{n}\left[(1 / \sigma) f\left(\left(a_{i}-\mathbf{x}_{i}^{T} \boldsymbol{\beta}\right) / \sigma\right)\right]^{k_{i}} \\
& \times\left[(1 / \sigma) f\left(\left(y_{i}-\mathbf{x}_{i}^{T} \boldsymbol{\beta}\right) / \sigma\right)\right]^{\ell_{i}} \\
& \prod_{i=1\left(i \neq i_{p}, \ldots, i_{\ell+p}\right)}^{n}(1 / \sigma) f\left(\left(y_{i}-\mathbf{x}_{i}^{T} \boldsymbol{\beta}\right) / \sigma\right) .
\end{aligned}
$$

In Step $a$, we use Lemma 1.1 to obtain $f(\omega) / f\left(y_{i}\right)=f\left(\left(y_{i}-a_{i}\right) / b_{i}\right) / f\left(y_{i}\right) \leq\left|b_{i}\right| D\left(\left|a_{i}\right|\right.$, $\left.\left|b_{i}\right|\right)$ for all $i \in \mathcal{I}_{\mathcal{O}}$. In Step $b$, we use $\pi(\boldsymbol{\beta}, \sigma) \leq B \max (1,1 / \sigma)=B / \sigma$. We also use that in any of the sets in which $\boldsymbol{\beta}$ can belong, there are at least $\ell+1$ nonoutlying points such that $\left|\mathbf{x}_{i}^{T} \boldsymbol{\beta}\right| \geq \omega / \gamma$ (corresponding to $\boldsymbol{\beta} \in \mathcal{F}_{i}^{c}$ for at least $\ell+1$ nonoutlying points). Indeed, the case in which there are the least nonoutliers such that $\left|\mathbf{x}_{i}^{T} \boldsymbol{\beta}\right| \geq$ $\omega / \gamma$ corresponds to $\boldsymbol{\beta} \in \cup_{i}\left(\mathcal{O}_{i} \cap \mathcal{F}_{i_{1}} \cap \cdots \cap \mathcal{F}_{i_{p-1}} \cap\left(\cap_{i_{p} \neq i_{1}, \ldots, i_{p-1}} \mathcal{F}_{i_{p}}^{c}\right)\right)$. In this case there are $p-1$ nonoutliers such that $\left|\mathbf{x}_{i}^{T} \boldsymbol{\beta}\right|<\omega / \gamma$ (say observations $i_{1}$ to $i_{p-1}$ ), which leaves at least $\ell+2 p-1-(p-1)$ nonoutliers such that $\left|\mathbf{x}_{i}^{T} \boldsymbol{\beta}\right| \geq \omega / \gamma$ (i.e. that there are $\ell+2 p-1-(p-1)$ sets in the intersection $\left.\cap_{i_{p} \neq i_{1}, \ldots, i_{p-1}} \mathcal{F}_{i_{p}}^{c}\right)$, and we know that $\ell+2 p-1-(p-1)=\ell+p>\ell+1$ because we only consider the models with $p \geq 2$. This implies that there exists a set of $\ell+1$ indices, say $\left\{i_{p}, \ldots, i_{\ell+p}\right\} \subset \mathcal{I}_{\mathcal{F}}$, such that for all $i \in\left\{i_{p}, \ldots, i_{\ell+p}\right\}$,

$$
f\left(\left(a_{i}-\mathbf{x}_{i}^{T} \boldsymbol{\beta}\right) / \sigma\right) \leq f(\omega /(2 \gamma \sigma)) \leq 2 \gamma D(0,2 \gamma) f(\omega / \sigma),
$$

using the monotonicity of the tails of $f$ in the first inequality because, if we define the constant $a_{(k)}:=\max _{i \in\{1, \ldots, k\}}\left|a_{i}\right|$ with $\omega \geq \mathrm{y} \geq(2 \gamma) a_{(k)}$, we have $\left|a_{i}-\mathbf{x}_{i}^{T} \boldsymbol{\beta}\right| / \sigma \geq$ $\left(\left|\mathbf{x}_{i}^{T} \boldsymbol{\beta}\right|-\left|a_{i}\right|\right) / \sigma \geq\left(\omega / \gamma-a_{(k)}\right) / \sigma \geq \omega /(2 \gamma \sigma) \geq \omega /(2 \gamma) \geq \mathrm{y} /(2 \gamma) \geq M$ if we choose $\mathrm{y} \geq 2 \gamma M$. In the second inequality, we use Lemma 1.1 (as mentioned in the proof of (1.4), we choose $\gamma \geq 2$ ). In Step $c$ above, we use the monotonicity of the tails of $|z| f(z)$ to obtain $(\omega / \sigma) f(\omega / \sigma) \leq \omega f(\omega)$ for $\ell$ terms, because $\omega / \sigma \geq \omega \geq \mathrm{y} \geq M$ if we choose $\mathrm{y} \geq M$. In Step $d$, we use $(1 / \sigma) f(\omega / \sigma) \leq B / \omega$. 
The integral of $(B / \omega)(1 / \sigma) \prod_{i=1\left(i \neq i_{p}, \ldots, i_{\ell+p}\right)}^{n}(1 / \sigma) f\left(\left(y_{i}-\mathbf{x}_{i}^{T} \boldsymbol{\beta}\right) / \sigma\right)$ is bounded by

$$
(B / \omega) \int_{\mathbb{R}^{p}} \int_{0}^{\infty}(1 / \sigma) \prod_{i=1\left(i \neq i_{p}, \ldots, i_{\ell+p}\right)}^{n}(1 / \sigma) f\left(\left(y_{i}-\mathbf{x}_{i}^{T} \boldsymbol{\beta}\right) / \sigma\right) d \sigma d \boldsymbol{\beta}=(B / \omega) m\left(\mathbf{y}_{\mathcal{I}_{R}}\right),
$$

where $m\left(\mathbf{y}_{\mathcal{I}_{R}}\right)$ is the marginal density arising from a prior proportional to $1 / \sigma$ and $n-(\ell+1)=k-1$ observations $\left(\mathbf{x}_{i}, y_{i}\right), i \in \mathcal{I}_{R}:=\{1, \ldots, n\} \backslash\left\{i_{p}, \ldots, i_{\ell+p}\right\}$. In order to prove that $(B / \omega) m\left(\mathbf{y}_{\mathcal{I}_{R}}\right) \rightarrow 0$ as $\omega \rightarrow \infty$, it suffices to prove that $m\left(\mathbf{y}_{\mathcal{I}_{R}}\right)$ is bounded by a constant that does not depend on $\omega$, because $1 / \omega \rightarrow 0$. In Section 1.1, we proved that a marginal, as $m\left(\mathbf{y}_{\mathcal{I}_{R}}\right)$, is bounded by a constant that does not depend on $\omega$ if the number of observations (which is $k-1$ in our case) is greater than or equal to $p+1$ if the prior divided by $1 / \sigma$ is bounded (which is the case for $m\left(\mathbf{y}_{\mathcal{I}_{R}}\right)$ ). Because we assume that $k \geq \ell+2 p-1$ and $\ell \geq 1$ (the proof for the case $\ell=0$ is trivial), and because we only consider the models with $p \geq 2, m\left(\mathbf{y}_{\mathcal{I}_{R}}\right)$ is the marginal of $k-1 \geq \ell+2 p-2 \geq p+1$ observations. As a result,

$$
(B / \omega) \int_{\mathbb{R}^{p}} \int_{0}^{\infty}(1 / \sigma) \prod_{i=1\left(i \neq i_{p}, \ldots, i_{\ell+p}\right)}^{n}(1 / \sigma) f\left(\left(y_{i}-\mathbf{x}_{i}^{T} \boldsymbol{\beta}\right) / \sigma\right) d \sigma d \boldsymbol{\beta} \rightarrow 0 \text { as } \omega \rightarrow \infty .
$$

We therefore have that

$$
\int_{\cup_{i} \mathcal{O}_{i}} \int_{0}^{1} \pi\left(\boldsymbol{\beta}, \sigma \mid \mathbf{y}_{\mathbf{k}}\right) \prod_{i=1}^{n}\left[\frac{(1 / \sigma) f\left(\left(y_{i}-\mathbf{x}_{i}^{T} \boldsymbol{\beta}\right) / \sigma\right)}{f\left(y_{i}\right)}\right]^{\ell_{i}} d \sigma d \boldsymbol{\beta} \rightarrow 0 \text { as } \omega \rightarrow \infty .
$$

Proof of Result (b). Consider $(\boldsymbol{\beta}, \sigma)$ such that $\pi(\boldsymbol{\beta}, \sigma)>0$ (the proof for the case $(\boldsymbol{\beta}, \sigma)$ such that $\pi(\boldsymbol{\beta}, \sigma)=0$ is trivial). We have, as $\omega \rightarrow \infty$,

$$
\begin{aligned}
\frac{\pi\left(\boldsymbol{\beta}, \sigma \mid \mathbf{y}_{\mathbf{n}}\right)}{\pi\left(\boldsymbol{\beta}, \sigma \mid \mathbf{y}_{\mathbf{k}}\right)} & =\frac{m\left(\mathbf{y}_{\mathbf{k}}\right)}{m\left(\mathbf{y}_{\mathbf{n}}\right)} \times \frac{\pi(\boldsymbol{\beta}, \sigma) \prod_{i=1}^{n}(1 / \sigma) f\left(\left(y_{i}-\mathbf{x}_{i}^{T} \boldsymbol{\beta}\right) / \sigma\right)}{\pi(\boldsymbol{\beta}, \sigma) \prod_{i=1}^{n}\left[(1 / \sigma) f\left(\left(y_{i}-\mathbf{x}_{i}^{T} \boldsymbol{\beta}\right) / \sigma\right)\right]^{k_{i}}} \\
& =\frac{m\left(\mathbf{y}_{\mathbf{k}}\right)}{m\left(\mathbf{y}_{\mathbf{n}}\right)} \prod_{i=1}^{n}\left[(1 / \sigma) f\left(\left(y_{i}-\mathbf{x}_{i}^{T} \boldsymbol{\beta}\right) / \sigma\right)\right]^{\ell_{i}} \\
& =\frac{m\left(\mathbf{y}_{\mathbf{k}}\right) \prod_{i=1}^{n}\left[f\left(y_{i}\right)\right]^{\ell_{i}}}{m\left(\mathbf{y}_{\mathbf{n}}\right)} \prod_{i=1}^{n}\left[\frac{(1 / \sigma) f\left(\left(y_{i}-\mathbf{x}_{i}^{T} \boldsymbol{\beta}\right) / \sigma\right)}{f\left(y_{i}\right)}\right]^{\ell_{i}} \rightarrow 1 .
\end{aligned}
$$

The first ratio in the last equality does not depend on $\boldsymbol{\beta}$ and $\sigma$ and converges towards 1 as $\omega \rightarrow \infty$ using Result (a). Also, the product converges towards 1 uniformly in any set $(\boldsymbol{\beta}, \sigma) \in[-\vartheta, \vartheta]^{p} \times[1 / \eta, \eta]$ using Proposition 2.2 in our paper given that $\mathbf{x}_{1}, \ldots, \mathbf{x}_{n}$ are fixed. Furthermore, since $f$ and $\pi(\boldsymbol{\beta}, \sigma) / \max (1,1 / \sigma)$ are bounded, $\pi\left(\boldsymbol{\beta}, \sigma \mid \mathbf{y}_{\mathbf{k}}\right)$ is also bounded on any set $(\boldsymbol{\beta}, \sigma) \in[-\eta, \eta]^{p} \times[1 / \eta, \eta]$. Then, we have

$$
\left|\pi\left(\boldsymbol{\beta}, \sigma \mid \mathbf{y}_{\mathbf{n}}\right)-\pi\left(\boldsymbol{\beta}, \sigma \mid \mathbf{y}_{\mathbf{k}}\right)\right|=\pi\left(\boldsymbol{\beta}, \sigma \mid \mathbf{y}_{\mathbf{k}}\right)\left|\frac{\pi\left(\boldsymbol{\beta}, \sigma \mid \mathbf{y}_{\mathbf{n}}\right)}{\pi\left(\boldsymbol{\beta}, \sigma \mid \mathbf{y}_{\mathbf{k}}\right)}-1\right| \rightarrow 0 \text { as } \omega \rightarrow \infty
$$


Proof of Result (c). Using Proposition 2.1 in our paper, we know that $\pi\left(\boldsymbol{\beta}, \sigma \mid \mathbf{y}_{\mathbf{k}}\right)$ and $\pi\left(\boldsymbol{\beta}, \sigma \mid \mathbf{y}_{\mathbf{n}}\right)$ are proper. Moreover, using Result (b), we have the pointwise convergence $\pi\left(\boldsymbol{\beta}, \sigma \mid \mathbf{y}_{\mathbf{n}}\right) \rightarrow \pi\left(\boldsymbol{\beta}, \sigma \mid \mathbf{y}_{\mathbf{k}}\right)$ as $\omega \rightarrow \infty$ for any $\boldsymbol{\beta} \in \mathbb{R}^{p}$ and $\sigma>0$, as a result of the uniform convergence. Then, the conditions of Scheffé's theorem (see Scheffé (1947)) are satisfied and we obtain the convergence in $L^{1}$ of $\pi\left(\boldsymbol{\beta}, \sigma \mid \mathbf{y}_{\mathbf{n}}\right)$ towards $\pi\left(\boldsymbol{\beta}, \sigma \mid \mathbf{y}_{\mathbf{k}}\right)$ as well as the following result:

$$
\lim _{\omega \rightarrow \infty} \int_{E} \pi\left(\boldsymbol{\beta}, \sigma \mid \mathbf{y}_{\mathbf{n}}\right) d \boldsymbol{\beta} d \sigma=\int_{E} \pi\left(\boldsymbol{\beta}, \sigma \mid \mathbf{y}_{\mathbf{k}}\right) d \boldsymbol{\beta} d \sigma
$$

uniformly for all sets $E \subset \mathbb{R}^{p} \times \mathbb{R}^{+}$. Result (c) follows directly.

Proof of Result (d). We prove that the moments converge through a mix of the strategies used to show Result (a) and that the moments exist in Proposition 2.1 in our paper. For any $M$, a positive integer, we have

$$
\begin{aligned}
\lim _{\omega \rightarrow \infty} \mathbb{E}\left[\sigma^{M} \mid \mathbf{y}_{\mathbf{n}}\right] & =\lim _{\omega \rightarrow \infty} \int_{0}^{\infty} \int_{\mathbb{R}^{p}} \sigma^{M} \pi\left(\boldsymbol{\beta}, \sigma \mid \mathbf{y}_{\mathbf{n}}\right) d \boldsymbol{\beta} d \sigma \\
& =\int_{0}^{\infty} \int_{\mathbb{R}^{p}} \lim _{\omega \rightarrow \infty} \sigma^{M} \pi\left(\boldsymbol{\beta}, \sigma \mid \mathbf{y}_{\mathbf{n}}\right) d \boldsymbol{\beta} d \sigma \\
& =\int_{0}^{\infty} \int_{\mathbb{R}^{p}} \sigma^{M} \pi\left(\boldsymbol{\beta}, \sigma \mid \mathbf{y}_{\mathbf{k}}\right) d \boldsymbol{\beta} d \sigma=\mathbb{E}\left[\sigma^{M} \mid \mathbf{y}_{\mathbf{k}}\right]
\end{aligned}
$$

assuming that we can interchange the limit and integral and using Result (b). To interchange the limit and integral, we again use Lebesgue's dominated convergence theorem which requires that the integrand is bounded by an integrable function of $\boldsymbol{\beta}$ and $\sigma$. We prove that it is the case using that

$$
\begin{aligned}
\sigma^{M} \pi\left(\boldsymbol{\beta}, \sigma \mid \mathbf{y}_{\mathbf{n}}\right)=\sigma^{M} & \frac{\pi(\boldsymbol{\beta}, \sigma) \prod_{i=1}^{n}\left[(1 / \sigma) f\left(\left(y_{i}-\mathbf{x}_{i}^{T} \boldsymbol{\beta}\right) / \sigma\right)\right]^{k_{i}}}{m\left(\mathbf{y}_{\mathbf{n}}\right)} \\
& \times \prod_{i=1}^{n}\left[\frac{(1 / \sigma) f\left(\left(y_{i}-\mathbf{x}_{i}^{T} \boldsymbol{\beta}\right) / \sigma\right)}{f\left(y_{i}\right)}\right]^{\ell_{i}} \prod_{i=1}^{n}\left[f\left(y_{i}\right)\right]^{\ell_{i}} .
\end{aligned}
$$

We have that $m\left(\mathbf{y}_{\mathbf{n}}\right)$ is bounded using Proposition 2.1 in our paper, $\prod_{i=1}^{n}\left[f\left(y_{i}\right)\right]^{\ell_{i}} \leq B^{\ell}$, and

$$
\sigma^{M} \prod_{i=1}^{n}\left[(1 / \sigma) f\left(\left(y_{i}-\mathbf{x}_{i}^{T} \boldsymbol{\beta}\right) / \sigma\right)\right]^{k_{i}} \leq B^{M} \prod_{i=M+1}^{n}\left[(1 / \sigma) f\left(\left(y_{i}-\mathbf{x}_{i}^{T} \boldsymbol{\beta}\right) / \sigma\right)\right]^{k_{i}},
$$

using $f \leq B$ for the $M$ first observations and assuming without loss of generality that these observations are nonoutliers (therefore $k_{1}=\ldots=k_{M}=1$ ). Therefore, we need to show that

$$
\pi(\boldsymbol{\beta}, \sigma) \prod_{i=M+1}^{n}\left[(1 / \sigma) f\left(\left(y_{i}-\mathbf{x}_{i}^{T} \boldsymbol{\beta}\right) / \sigma\right)\right]^{k_{i}} \prod_{i=1}^{n}\left[\frac{(1 / \sigma) f\left(\left(y_{i}-\mathbf{x}_{i}^{T} \boldsymbol{\beta}\right) / \sigma\right)}{f\left(y_{i}\right)}\right]^{\ell_{i}}
$$




$$
=m\left(\mathbf{y}_{\mathbf{k}}^{*}\right) \pi\left(\boldsymbol{\beta}, \sigma \mid \mathbf{y}_{\mathbf{k}}^{*}\right) \prod_{i=1}^{n}\left[\frac{(1 / \sigma) f\left(\left(y_{i}-\mathbf{x}_{i}^{T} \boldsymbol{\beta}\right) / \sigma\right)}{f\left(y_{i}\right)}\right]^{\ell_{i}}
$$

is bounded by an integrable function of $\boldsymbol{\beta}$ and $\sigma$, where $\mathbf{y}_{\mathbf{k}}^{*}:=\mathbf{y}_{\mathbf{k}} \backslash\left\{y_{1}, \ldots, y_{M}\right\}$ (the nonoutlier group without the first $M$ nonoutliers). In the proof of Result (a), it has been shown that it is the case under the assumptions of Theorem 2.1 in our paper, which are satisfied considering this modified data set with $k-M \geq n / 2+(p-1 / 2)$ (see the additional assumption for Result (d) of Theorem 2.1 in our paper).

For the expectations $\mathbb{E}\left[\beta_{j}^{M} \mid \mathbf{y}_{\mathbf{n}}\right]$, we proceed in the same way, we simply additionally consider that, as in the proof of Proposition 2.1 (see Section 1.1), $\beta_{j}$ can be rewritten as $\mathbf{e}_{j}^{T} \boldsymbol{\beta}$, and that next, $\mathbf{e}_{j}$ can be expressed as a linear combination of $p$ vectors $\mathbf{x}_{i_{1}}, \ldots, \mathbf{x}_{i_{p}}$, where now these are selected among the nonoutliers, i.e. $i_{1}, \ldots, i_{p} \in\left\{i: k_{i}=1\right\}$. We detail the case $M=1$. From it and what has been done before, it will be clear the result holds in general, with further technicalities. As above,

$$
\begin{aligned}
\lim _{\omega \rightarrow \infty} \mathbb{E}\left[\beta_{j} \mid \mathbf{y}_{\mathbf{n}}\right] & =\lim _{\omega \rightarrow \infty} \int_{0}^{\infty} \int_{\mathbb{R}^{p}} \beta_{j} \pi\left(\boldsymbol{\beta}, \sigma \mid \mathbf{y}_{\mathbf{n}}\right) d \boldsymbol{\beta} d \sigma \\
& =\int_{0}^{\infty} \int_{\mathbb{R}^{p}} \lim _{\omega \rightarrow \infty} \beta_{j} \pi\left(\boldsymbol{\beta}, \sigma \mid \mathbf{y}_{\mathbf{n}}\right) d \boldsymbol{\beta} d \sigma \\
& =\int_{0}^{\infty} \int_{\mathbb{R}^{p}} \beta_{j} \pi\left(\boldsymbol{\beta}, \sigma \mid \mathbf{y}_{\mathbf{k}}\right) d \boldsymbol{\beta} d \sigma=\mathbb{E}\left[\beta_{j} \mid \mathbf{y}_{\mathbf{k}}\right],
\end{aligned}
$$

assuming that we can interchange the limit and integral and using Result (b). As above, we have to show that the integrand is bounded above by an integrable function of $\boldsymbol{\beta}$ and $\sigma$. We beforehand use that

$$
\beta_{j}=\mathbf{e}_{j}^{T} \boldsymbol{\beta}=\sum_{s=1}^{p} a_{s} \mathbf{x}_{i_{s}}^{T} \boldsymbol{\beta}=\sum_{s=1}^{p} a_{s}\left(y_{i_{s}}-\mathbf{x}_{i_{s}}^{T} \boldsymbol{\beta}\right)-\sum_{s=1}^{p} a_{s} y_{i_{s}}
$$

as mentioned, where $a_{1}, \ldots, a_{p} \in \mathbb{R}$ and $i_{1}, \ldots, i_{p} \in\left\{i: k_{i}=1\right\}$. The integrand thus becomes a sum of $2 p$ terms, and we prove that each one of them is bounded above by an integrable function of $\boldsymbol{\beta}$ and $\sigma$, which will complete the proof. As above

$$
\begin{gathered}
a_{s}\left(y_{i_{s}}-\mathbf{x}_{i_{s}}^{T} \boldsymbol{\beta}\right) \pi\left(\boldsymbol{\beta}, \sigma \mid \mathbf{y}_{\mathbf{n}}\right) \leq\left|a_{s}\right|\left|y_{i_{s}}-\mathbf{x}_{i_{s}}^{T} \boldsymbol{\beta}\right| \frac{\pi(\boldsymbol{\beta}, \sigma) \prod_{i=1}^{n}\left[(1 / \sigma) f\left(\left(y_{i}-\mathbf{x}_{i}^{T} \boldsymbol{\beta}\right) / \sigma\right)\right]^{k_{i}}}{m\left(\mathbf{y}_{\mathbf{n}}\right)} \\
\times \prod_{i=1}^{n}\left[\frac{(1 / \sigma) f\left(\left(y_{i}-\mathbf{x}_{i}^{T} \boldsymbol{\beta}\right) / \sigma\right)}{f\left(y_{i}\right)}\right]^{\ell_{i}} \prod_{i=1}^{n}\left[f\left(y_{i}\right)\right]^{\ell_{i}} .
\end{gathered}
$$

We have that $m\left(\mathbf{y}_{\mathbf{n}}\right)$ is bounded using Proposition 2.1 in our paper, $\prod_{i=1}^{n}\left[f\left(y_{i}\right)\right]^{\ell_{i}} \leq B^{\ell}$, and

$$
\left|y_{i_{s}}-\mathbf{x}_{i_{s}}^{T} \boldsymbol{\beta}\right| \prod_{i=1}^{n}\left[(1 / \sigma) f\left(\left(y_{i}-\mathbf{x}_{i}^{T} \boldsymbol{\beta}\right) / \sigma\right)\right]^{k_{i}} \leq B \prod_{i=1\left(i \neq i_{s}\right)}^{n}\left[(1 / \sigma) f\left(\left(y_{i}-\mathbf{x}_{i}^{T} \boldsymbol{\beta}\right) / \sigma\right)\right]^{k_{i}}
$$

using

$$
\left(\left|y_{i_{s}}-\mathbf{x}_{i_{s}}^{T} \boldsymbol{\beta}\right| / \sigma\right) f\left(\left(y_{i_{s}}-\mathbf{x}_{i_{s}}^{T} \boldsymbol{\beta}\right) / \sigma\right) \leq B
$$


Therefore, $a_{s}\left(y_{i_{s}}-\mathbf{x}_{i_{s}}^{T} \boldsymbol{\beta}\right) \pi\left(\boldsymbol{\beta}, \sigma \mid \mathbf{y}_{\mathbf{n}}\right)$ is bounded above by a constant times

$$
\begin{gathered}
\pi(\boldsymbol{\beta}, \sigma) \prod_{i=1\left(i \neq i_{s}\right)}^{n}\left[(1 / \sigma) f\left(\left(y_{i}-\mathbf{x}_{i}^{T} \boldsymbol{\beta}\right) / \sigma\right)\right]^{k_{i}} \prod_{i=1}^{n}\left[\frac{(1 / \sigma) f\left(\left(y_{i}-\mathbf{x}_{i}^{T} \boldsymbol{\beta}\right) / \sigma\right)}{f\left(y_{i}\right)}\right]^{\ell_{i}} \\
=m\left(\mathbf{y}_{\mathbf{k}}^{*}\right) \pi\left(\boldsymbol{\beta}, \sigma \mid \mathbf{y}_{\mathbf{k}}^{*}\right) \prod_{i=1}^{n}\left[\frac{(1 / \sigma) f\left(\left(y_{i}-\mathbf{x}_{i}^{T} \boldsymbol{\beta}\right) / \sigma\right)}{f\left(y_{i}\right)}\right]^{\ell_{i}}
\end{gathered}
$$

where $\mathbf{y}_{\mathbf{k}}^{*}:=\mathbf{y}_{\mathbf{k}} \backslash\left\{y_{i_{s}}\right\}$ (the nonoutlier group without the $i_{s}$-th nonoutlier). As mentioned above, in the proof of Result (a), it has been shown that it is the case under the assumptions of Theorem 2.1 in our paper, which are satisfied considering this modified data set with $k-1 \geq n / 2+(p-1 / 2)$ (see the additional assumption for Result (d) of Theorem 2.1 in our paper $)$. The proofs for the terms with $a_{s} y_{i_{s}} \pi\left(\boldsymbol{\beta}, \sigma \mid \mathbf{y}_{\mathbf{n}}\right)$ is similar.

\section{Complement of Section 3.2 in our Paper}

In Section 3.2 in our paper, we mention that the first derivative of the divergence

$$
\mathrm{KL}(\boldsymbol{\beta}, \sigma):=\int \log \left(g\left(y_{i}\right) / p_{(\boldsymbol{\beta}, \sigma)}\left(y_{i}\right)\right) g\left(y_{i}\right) d y_{i}
$$

with respect to $\boldsymbol{\beta}$ equals 0 at $\boldsymbol{\beta}_{0}$, and this for any value of $\sigma$. We also mention that while setting $\boldsymbol{\beta}=\boldsymbol{\beta}_{0}$ in (2.1), it is minimised at $\sigma^{*}$ which depends on $\rho$. Finally, we mention that most of the regularity conditions in Bunke et al. (1998) are satisfied. We now show all this. We rewrite the divergence:

$$
\mathrm{KL}(\boldsymbol{\theta})=\mathbb{E}_{g}[\log g(Y)]-\mathbb{E}_{g}\left[\log p_{\boldsymbol{\theta}}(Y)\right],
$$

where $\mathbb{E}_{g}$ denotes the expectation with respect to $g$, and omitting the index $i$. The first term is computed exactly:

$\mathbb{E}_{g}[\log g(Y)]=-\frac{1}{2} \log (2 \pi)-\log \sigma_{0}-\frac{1}{2 \sigma_{0}^{2}} \mathbb{E}_{g}\left[\left(Y-\mathbf{x}^{T} \boldsymbol{\beta}_{0}\right)^{2}\right]=-\frac{1}{2}(\log (2 \pi)-1)-\log \sigma_{0}$.

The second term is rewritten as:

$$
\begin{aligned}
\mathbb{E}_{g}\left[\log p_{\boldsymbol{\theta}}(Y)\right] & =\int\left(\log f\left(\frac{y-\mathbf{x}^{T} \boldsymbol{\beta}}{\sigma}\right)-\log \sigma\right) \frac{1}{\sqrt{2 \pi} \sigma_{0}} \exp \left(-\frac{1}{2 \sigma_{0}^{2}}\left(y-\mathbf{x}^{T} \boldsymbol{\beta}_{0}\right)^{2}\right) d y \\
& =\int \log f(z \eta+\delta \eta) \varphi(z) d z+\log \eta-\log \sigma_{0}
\end{aligned}
$$

using the change of variable $z=\left(y-\mathbf{x}^{T} \boldsymbol{\beta}_{0}\right) / \sigma_{0}$, and denoting $\delta:=\mathbf{x}^{T}\left(\boldsymbol{\beta}_{0}-\boldsymbol{\beta}\right) / \sigma_{0}$ and $\eta:=\sigma_{0} / \sigma$. Therefore, minimising the divergence is equivalent to maximising

$$
\int \log f(z \eta+\delta \eta) \varphi(z) d z+\log \eta
$$


We now show that we can interchange the derivative with respect to $\delta$ and the integral. The first derivative of $f$ is given by

$$
f^{\prime}(z)=\left\{\begin{array}{l}
-z \varphi(z) \quad \text { if } \quad|z|<\tau \\
-\varphi(\tau) \tau(\log \tau)^{\lambda+1} \frac{\operatorname{sign}(z)}{z^{2}} \frac{1}{(\log |z|)^{\lambda+1}}\left(1+\frac{\lambda+1}{\log |z|}\right) \quad \text { if } \quad|z|>\tau, \\
\text { does not exist if } z \text { equals }-\tau \text { or } \tau
\end{array}\right.
$$

where $\operatorname{sign}(\cdot)$ is the sign function. For completeness, we assign the values $-z \varphi(z)$ to $f^{\prime}(z)$ when $|z|=\tau$. Note that we are allowed to do this because these points have null measure. We thus consider that

$$
\frac{f^{\prime}(z)}{f(z)}=\left\{\begin{array}{ll}
-z & \text { if } \quad|z| \leq \tau \\
-\frac{\operatorname{sign}(z)}{|z|}\left(1+\frac{\lambda+1}{\log |z|}\right)
\end{array} \quad \text { if } \quad|z|>\tau .\right.
$$

This function is bounded. Consequently,

$$
\frac{\partial}{\partial \delta} \log f(z \eta+\delta \eta)=\eta \frac{f^{\prime}(z \eta+\delta \eta)}{f(z \eta+\delta \eta)}
$$

is bounded for any value of $\eta$, which implies that we can interchange the derivative and the integral. If $\delta=0$, the integral is equal to 0 , because $f^{\prime}(-z) / f(z)=-f^{\prime}(z) / f(z)$, and

$$
\begin{aligned}
\int \frac{f^{\prime}(z \eta)}{f(z \eta)} \varphi(z) d z & =\int_{-\infty}^{0} \frac{f^{\prime}(z \eta)}{f(z \eta)} \varphi(z) d z+\int_{0}^{\infty} \frac{f^{\prime}(z \eta)}{f(z \eta)} \varphi(z) d z \\
& =\int_{0}^{\infty} \frac{f^{\prime}(-z \eta)}{f(-z \eta)} \varphi(-z) d z+\int_{0}^{\infty} \frac{f^{\prime}(z \eta)}{f(z \eta)} \varphi(z) d z \\
& =-\int_{0}^{\infty} \frac{f^{\prime}(z \eta)}{f(z \eta)} \varphi(z) d z+\int_{0}^{\infty} \frac{f^{\prime}(z \eta)}{f(z \eta)} \varphi(z) d z
\end{aligned}
$$

Notice that this is true for any value of $\eta$. Analysing the second derivative may allow to rigorously conclude that the divergence is (uniquely) minimised with respect to $\boldsymbol{\beta}$ at $\boldsymbol{\beta}_{0}$. If it is strictly negative for any value of $\eta$, it is the case. We now analyse

$$
\int \log f(z \eta) \varphi(z) d z+\log \eta
$$

In the same way as for $\delta$, we show that we can interchange the derivative with respect to $\eta$ and the integral. We have

$$
\frac{\partial}{\partial \eta} \log f(z \eta)=z \frac{f^{\prime}(z \eta)}{f(z \eta)}
$$

which is bounded by $|z|$ times a constant. This is an integrable function with respect to $\varphi$. Therefore, we can interchange the integral and the derivative:

$$
\frac{\partial}{\partial \eta}\left(\int \log f(z \eta) \varphi(z) d z+\log \eta\right)=\int z \frac{f^{\prime}(z \eta)}{f(z \eta)} \varphi(z) d z+\frac{1}{\eta} .
$$


Setting the derivative equals to 0 leads to

$$
\int z \eta \frac{f^{\prime}(z \eta)}{f(z \eta)} \varphi(z) d z=-1
$$

We cannot solve this explicitly, but numerical calculations show that the solution is unique. For instance, (2.2) as a function of $\eta$ with $\rho=0.95$ is shown in Figure 1 (a), with the maximiser $\eta^{*}$ as a function of $\rho$ in Figure 1 (b). The previous analysis suggests that $\left(\boldsymbol{\beta}^{*}, \sigma^{*}\right)=\left(\boldsymbol{\beta}_{0}, \sigma_{0} / \eta^{*}\right)$.

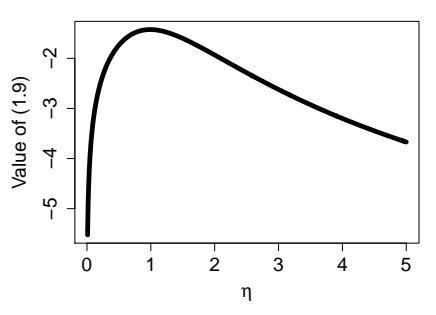

(a)

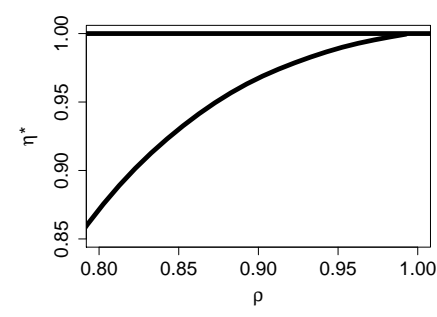

(b)

Figure 1: (a) Value of (2.2) as a function of $\eta$ with $\rho=0.95$; (b) maximiser $\eta^{*}$ as a function of $\rho$

We now show that most of the regularity conditions in Bunke et al. (1998) are satisfied.

Condition 1. The parameter space $\boldsymbol{\Theta}$ is a closed (possibly unbounded) convex set in $\mathbb{R}^{d}$ with a nonempty interior. The density $p_{\boldsymbol{\theta}}(y)$ is bounded for all $\boldsymbol{\theta}$ and $y$, and its carrier $\left\{y: p_{\boldsymbol{\theta}}(y)>0\right\}$ is the same for all $\boldsymbol{\theta}$.

This condition is not directly satisfied because the parameter space is open $(\sigma>$ 0 ). But it should not be a problem if we can show that it is possible to choose $\epsilon>0$ such $\sigma_{0} \in[\epsilon, \infty)$ and that the mass outside of this set goes to 0 as the sample size increases. Indeed, we could "define" the parameter space to be $[\epsilon, \infty) \times \mathbb{R}^{p}$ which is a closed convex set and lose nothing asymptotically. On this parameter space $p_{\boldsymbol{\theta}}(y)$ is bounded for all $\boldsymbol{\theta}$ and $y$, and its carrier $\left\{y: p_{\boldsymbol{\theta}}(y)>0\right\}=\mathbb{R}$ is the same for all $\boldsymbol{\theta}$.

Condition 2. For all $\boldsymbol{\theta}$, there is a sphere $S[\boldsymbol{\theta}, r]$ of center $\boldsymbol{\theta}$ and radius $r$ with

$$
\mathbb{E}_{g}\left[\sup \left\{\left|\log \left[g(Y) / p_{t}(Y)\right]\right|: t \in S[\boldsymbol{\theta}, r]\right\}\right],
$$

where $\mathbb{E}_{g}$ is the expectation with respect to $g$.

Lemma 1.1 implies that

$$
\frac{p_{\left(\boldsymbol{\beta}_{0}, \sigma_{0}\right)}(y)}{p_{t}(y)}
$$


is bounded both from below and above on $S[\boldsymbol{\theta}, r]$. Also, $g(y) / p_{\left(\boldsymbol{\beta}_{0}, \sigma_{0}\right)}(y)$ does not depend on $t$. Therefore, there exists a positive constant $c$ such that

$$
\begin{aligned}
\mathbb{E}_{g}\left[\sup \left\{\left|\log \left[g(Y) / p_{t}(Y)\right]\right|: t \in S[\boldsymbol{\theta}, r]\right\}\right] & \leq c \mathbb{E}_{g}\left[\left|\log \left[g(Y) / p_{\left(\boldsymbol{\beta}_{0}, \sigma_{0}\right)}(Y)\right]\right|\right] \\
& =c \mathbb{E}_{\varphi}[|\log [\varphi(Z) / f(Z)]|],
\end{aligned}
$$

where a change of variables has been used in the last equality and $f$ is the LPTN density. We also have that

$$
\frac{\varphi(z)}{f(z)}=\left\{\begin{array}{l}
1 \quad \text { if } \quad|z| \leq \tau \\
c_{2} \exp \left(-z^{2} / 2\right)|z|(\log |z|)^{\lambda+1} \quad \text { if } \quad|z|>\tau
\end{array}\right.
$$

where $c_{2}$ is a positive constant. Consequently,

$$
\begin{aligned}
& c \mathbb{E}_{\varphi}[|\log [\varphi(Z) / f(Z)]|]=c \mathbb{E}_{\varphi}[|\log [\varphi(Z) / f(Z)]| \mathbb{1}(|Z|>\tau)] \\
& \leq c \log \left(c_{2}\right)+c \mathbb{E}_{\varphi}\left[\left(Z^{2} / 2+\left|\log \left(|Z|(\log |Z|)^{\lambda+1}\right)\right|\right) \mathbb{1}(|Z|>\tau)\right]<\infty .
\end{aligned}
$$

Condition 3. For all fixed $y$, the density $p_{\boldsymbol{\theta}}(y)$ has a continuous derivative $p_{\boldsymbol{\theta}}^{\prime}(y)$ with respect to $\boldsymbol{\theta}$ and there are positive constants $c, b_{0}$ such that

$$
\int\left\|\left[p_{\boldsymbol{\theta}}(y)\right]^{-1} p_{\boldsymbol{\theta}}^{\prime}(y)\right\|^{4(d+1)} p_{\boldsymbol{\theta}}(y) d y<c\left(1+\|\boldsymbol{\theta}\|^{b_{0}}\right),
$$

for all $\boldsymbol{\theta}$, where $\|\cdot\|$ denotes a norm in $\mathbb{R}^{d}$.

In our case, the density $p_{\boldsymbol{\theta}}(y)$ has an almost everywhere continuous derivative. We believe this should not cause fundamental problems for rigorously prove the result.

We have that

$$
\frac{\frac{\partial}{\partial \boldsymbol{\beta}}\left(\frac{1}{\sigma} f\left(\frac{y-\mathbf{x}^{T} \boldsymbol{\beta}}{\sigma}\right)\right)}{\frac{1}{\sigma} f\left(\frac{y-\mathbf{x}^{T} \boldsymbol{\beta}}{\sigma}\right)}=-\frac{\mathbf{x}^{T}}{\sigma} \frac{f^{\prime}\left(\frac{y-\mathbf{x}^{T} \boldsymbol{\beta}}{\sigma}\right)}{f\left(\frac{y-\mathbf{x}^{T} \boldsymbol{\beta}}{\sigma}\right)}=-\frac{\mathbf{x}^{T}}{\sigma} \frac{f^{\prime}(z)}{f(z)},
$$

and

$$
\frac{\frac{\partial}{\partial \sigma}\left(\frac{1}{\sigma} f\left(\frac{y-\mathbf{x}^{T} \boldsymbol{\beta}}{\sigma}\right)\right)}{\frac{1}{\sigma} f\left(\frac{y-\mathbf{x}^{T} \boldsymbol{\beta}}{\sigma}\right)}=-\frac{1}{\sigma} \frac{f\left(\frac{y-\mathbf{x}^{T} \boldsymbol{\beta}}{\sigma}\right)}{f\left(\frac{y-\mathbf{x}^{T} \boldsymbol{\beta}}{\sigma}\right)}-\frac{y-\mathbf{x}^{T} \boldsymbol{\beta}}{\sigma^{2}} \frac{f^{\prime}\left(\frac{y-\mathbf{x}^{T} \boldsymbol{\beta}}{\sigma}\right)}{f\left(\frac{y-\mathbf{x}^{T} \boldsymbol{\beta}}{\sigma}\right)}=-\frac{1}{\sigma}\left(1+\frac{f^{\prime}(z)}{f(z)}\right),
$$

after the change of variable $z=\left(y-\mathbf{x}^{T} \boldsymbol{\beta}\right) / \sigma$. We use the traditional Euclidian norm. The function $f^{\prime} / f$ is bounded. Therefore,

$$
\int\left\|\left[p_{\boldsymbol{\theta}}(y)\right]^{-1} p_{\boldsymbol{\theta}}^{\prime}(y)\right\|^{4(d+1)} p_{\boldsymbol{\theta}}(y) d y \leq c_{0} \frac{1}{\sigma^{4(p+2)}},
$$

where $c_{0}$ is a positive constant. If the parameter space is $[\epsilon, \infty) \times \mathbb{R}^{p}$, it is easily seen that

$$
c_{0} \frac{1}{\sigma^{4(p+2)}}<c\left(1+\|\boldsymbol{\theta}\|^{b_{0}}\right) .
$$


Condition 4. For some positive constant $b_{1}$ the affinity

$$
\varrho(\boldsymbol{\theta}):=\int\left[p_{\boldsymbol{\theta}}(y) g(y)\right]^{1 / 2} d y
$$

has the behaviour

$$
\varrho(\boldsymbol{\theta})<c\|\boldsymbol{\theta}\|^{-b_{1}}, \quad \boldsymbol{\theta} \in \boldsymbol{\Theta} .
$$

Condition 4 is, in our opinion, the condition that will require a careful analysis.

Condition 5. There are positive constants $b_{2}, b_{3}$ so that for all $\boldsymbol{\theta}$ and $r>0$ it holds that

$$
\pi(S[\boldsymbol{\theta}, r]) \leq c r^{b_{2}}\left(1+(\|\boldsymbol{\theta}\|+r)^{b_{3}}\right),
$$

where $\pi(S[\boldsymbol{\theta}, r])$ measure of $S[\boldsymbol{\theta}, r]$ under the prior. Moreover, $\pi(S[\boldsymbol{\theta}, r])>0$ for all $r>0$ and $\boldsymbol{\theta}$.

The last part is satisfied if the prior is strictly positive over the parameter space, which is usually the case (it is true in our numerical analyses). The first part essentially requires that the measure does not "explode" in some areas. Under the assumption mentioned in Section 2.1 in our paper on the prior and if the parameter space is $[\epsilon, \infty) \times \mathbb{R}^{p}$, we have that

$$
\pi(S[\boldsymbol{\theta}, r])=\int \mathbb{1}_{S[\boldsymbol{\theta}, r]} \pi(\boldsymbol{\theta}) d \boldsymbol{\theta} \leq \frac{1}{\epsilon} \int \mathbb{1}_{S[\boldsymbol{\theta}, r]} d \boldsymbol{\theta}=\frac{c}{\epsilon} r^{p+1},
$$

implying that the first part holds.

Condition 6. Let $L: \boldsymbol{\Theta} \times \boldsymbol{\Theta} \rightarrow \mathbb{R}^{+}$be a measurable loss function with $L(\boldsymbol{\theta}, \boldsymbol{\theta})=0$, $c_{1}, c_{2}, c_{3}, b_{4}, b_{5}$ be positive constants such that

$$
\left(c_{1}\|t-\boldsymbol{\theta}\|^{b_{4}}\right) \wedge c_{2} \leq L(t, \boldsymbol{\theta}) \leq c_{3}\|t-\boldsymbol{\theta}\|^{b_{5}},
$$

for all $t, \boldsymbol{\theta} \in \mathbf{\Theta}$.

It is easily seen that the quadratic loss function satisfies this, pointing towards the consistency of the posterior mean of $\boldsymbol{\beta}$. Under Conditions 1 to 5 , a result in Bunke et al. (1998) indicates that the posterior density concentrates around $\boldsymbol{\theta}^{*}=\left(\boldsymbol{\beta}_{0}, \sigma^{*}\right)$, pointing in this case towards the consistency of the part of the posterior mode associated with $\boldsymbol{\beta}$.

\section{Other Result}

Proposition 3.1. If $f=\mathcal{N}(0,1)$ and $\pi(\boldsymbol{\beta}, \sigma) \propto \pi(\sigma) \times 1$, then

$$
\boldsymbol{\beta} \mid \sigma, \mathbf{y}_{\mathbf{n}} \sim \mathcal{N}\left(\left(\mathbf{X}_{\mathbf{n}}{ }^{T} \mathbf{X}_{\mathbf{n}}\right)^{-1} \mathbf{X}_{\mathbf{n}}{ }^{T} \mathbf{y}_{\mathbf{n}}, \sigma^{2}\left(\mathbf{X}_{\mathbf{n}}{ }^{T} \mathbf{X}_{\mathbf{n}}\right)^{-1}\right),
$$

and

$$
\pi\left(\sigma \mid \mathbf{y}_{\mathbf{n}}\right) \propto \pi(\sigma) \frac{1}{\sigma^{n-p}} \exp \left(-\frac{1}{2 \sigma^{2}}\left\|\mathbf{y}_{\mathbf{n}}-\hat{\mathbf{y}}_{\mathbf{n}}\right\|^{2}\right)
$$


where $\mathbf{X}_{\mathbf{n}}$ is matrix whose rows are given by $\mathbf{x}_{1}^{T}, \ldots, \mathbf{x}_{n}^{T}, \hat{\mathbf{y}}_{\mathbf{n}}:=\mathbf{X}_{\mathbf{n}}\left(\mathbf{X}_{\mathbf{n}}{ }^{T} \mathbf{X}_{\mathbf{n}}\right)^{-1} \mathbf{X}_{\mathbf{n}}{ }^{T} \mathbf{y}_{\mathbf{n}}$, and $\|\cdot\|$ is the Euclidean norm. In particular, if $\pi(\sigma) \propto 1 / \sigma, \sigma^{2} \mid \mathbf{y}_{\mathbf{n}} \sim$ Inverse $-\Gamma((n-$ p) $\left./ 2,\left\|\mathbf{y}_{\mathbf{n}}-\hat{\mathbf{y}}_{\mathbf{n}}\right\|^{2} / 2\right)$.

Proof. The proof relies essentially on straightforward calculations. We have

$$
\pi\left(\boldsymbol{\beta}, \sigma \mid \mathbf{y}_{\mathbf{n}}\right) \propto \pi(\sigma) \prod_{i=1}^{n} \frac{1}{\sqrt{2 \pi} \sigma} \exp \left(-\frac{1}{2 \sigma^{2}}\left(y_{i}-\mathbf{x}_{i}^{T} \boldsymbol{\beta}\right)^{2}\right) .
$$

We therefore have sum of squares in the exponential and we first analyse it. We have

$$
\sum_{i=1}^{n}\left(y_{i}-\mathbf{x}_{i}^{T} \boldsymbol{\beta}\right)^{2}=\sum_{i=1}^{n}\left(y_{i}^{2}-2 y_{i} \mathbf{x}_{i}^{T} \boldsymbol{\beta}+\left(\mathbf{x}_{i}^{T} \boldsymbol{\beta}\right)^{2}\right) .
$$

We analyse the middle term:

$$
-2 \sum_{i=1}^{n} y_{i} \mathbf{x}_{i}^{T} \boldsymbol{\beta}=-2 \boldsymbol{\beta}^{T} \sum_{i=1}^{n} \mathbf{x}_{i} y_{i}=-2 \boldsymbol{\beta}^{T} \mathbf{X}_{\mathbf{n}}{ }^{T} \mathbf{y}_{\mathbf{n}}=-2\left(\mathbf{X}_{\mathbf{n}}{ }^{T} \mathbf{y}_{\mathbf{n}}\right)^{T} \boldsymbol{\beta} .
$$

The last term is such that

$$
\sum_{i=1}^{n} \mathbf{x}_{i}^{T} \boldsymbol{\beta} \mathbf{x}_{i}^{T} \boldsymbol{\beta}=\sum_{i=1}^{n} \boldsymbol{\beta}^{T} \mathbf{x}_{i} \mathbf{x}_{i}^{T} \boldsymbol{\beta}=\boldsymbol{\beta}^{T} \mathbf{X}_{\mathbf{n}}^{T} \mathbf{X}_{\mathbf{n}} \boldsymbol{\beta}
$$

Adding and subtracting $\left(\mathbf{X}_{\mathbf{n}}{ }^{T} \mathbf{X}_{\mathbf{n}}\right)^{-1} \mathbf{X}_{\mathbf{n}}{ }^{T} \mathbf{y}_{\mathbf{n}}$ to $\boldsymbol{\beta}$ before the first transpose yields

$$
\begin{aligned}
(\boldsymbol{\beta}- & \left.\left(\mathbf{X}_{\mathbf{n}}{ }^{T} \mathbf{X}_{\mathbf{n}}\right)^{-1} \mathbf{X}_{\mathbf{n}}{ }^{T} \mathbf{y}_{\mathbf{n}}+\left(\mathbf{X}_{\mathbf{n}}{ }^{T} \mathbf{X}_{\mathbf{n}}\right)^{-1} \mathbf{X}_{\mathbf{n}}{ }^{T} \mathbf{y}_{\mathbf{n}}\right)^{T} \mathbf{X}_{\mathbf{n}}{ }^{T} \mathbf{X}_{\mathbf{n}} \boldsymbol{\beta} \\
& =\left(\boldsymbol{\beta}-\left(\mathbf{X}_{\mathbf{n}}{ }^{T} \mathbf{X}_{\mathbf{n}}\right)^{-1} \mathbf{X}_{\mathbf{n}}{ }^{T} \mathbf{y}_{\mathbf{n}}\right)^{T} \mathbf{X}_{\mathbf{n}}{ }^{T} \mathbf{X}_{\mathbf{n}} \boldsymbol{\beta}+\left(\mathbf{X}_{\mathbf{n}}{ }^{T} \mathbf{y}_{\mathbf{n}}\right)^{T}\left(\mathbf{X}_{\mathbf{n}}{ }^{T} \mathbf{X}_{\mathbf{n}}\right)^{-1} \mathbf{X}_{\mathbf{n}}{ }^{T} \mathbf{X}_{\mathbf{n}} \boldsymbol{\beta} .
\end{aligned}
$$

The last term on the RHS cancels out with one in (3.1). We again add and subtract $\left(\mathbf{X}_{\mathbf{n}}{ }^{T} \mathbf{X}_{\mathbf{n}}\right)^{-1} \mathbf{X}_{\mathbf{n}}{ }^{T} \mathbf{y}_{\mathbf{n}}$ to $\boldsymbol{\beta}$ :

$$
\begin{gathered}
\left(\boldsymbol{\beta}-\left(\mathbf{X}_{\mathbf{n}}{ }^{T} \mathbf{X}_{\mathbf{n}}\right)^{-1} \mathbf{X}_{\mathbf{n}}{ }^{T} \mathbf{y}_{\mathbf{n}}\right)^{T} \mathbf{X}_{\mathbf{n}}{ }^{T} \mathbf{X}_{\mathbf{n}}\left(\boldsymbol{\beta}-\left(\mathbf{X}_{\mathbf{n}}{ }^{T} \mathbf{X}_{\mathbf{n}}\right)^{-1} \mathbf{X}_{\mathbf{n}}{ }^{T} \mathbf{y}_{\mathbf{n}}+\left(\mathbf{X}_{\mathbf{n}}{ }^{T} \mathbf{X}_{\mathbf{n}}\right)^{-1} \mathbf{X}_{\mathbf{n}}{ }^{T} \mathbf{y}_{\mathbf{n}}\right) \\
=\left(\boldsymbol{\beta}-\left(\mathbf{X}_{\mathbf{n}}{ }^{T} \mathbf{X}_{\mathbf{n}}\right)^{-1} \mathbf{X}_{\mathbf{n}}{ }^{T} \mathbf{y}_{\mathbf{n}}\right)^{T} \mathbf{X}_{\mathbf{n}}{ }^{T} \mathbf{X}_{\mathbf{n}}\left(\boldsymbol{\beta}-\left(\mathbf{X}_{\mathbf{n}}{ }^{T} \mathbf{X}_{\mathbf{n}}\right)^{-1} \mathbf{X}_{\mathbf{n}}{ }^{T} \mathbf{y}_{\mathbf{n}}\right) \\
\quad+\left(\boldsymbol{\beta}-\left(\mathbf{X}_{\mathbf{n}}{ }^{T} \mathbf{X}_{\mathbf{n}}\right)^{-1} \mathbf{X}_{\mathbf{n}}{ }^{T} \mathbf{y}_{\mathbf{n}}\right)^{T} \mathbf{X}_{\mathbf{n}}{ }^{T} \mathbf{y}_{\mathbf{n}} .
\end{gathered}
$$

The last term on the RHS is equal to $\boldsymbol{\beta}^{T} \mathbf{X}_{\mathbf{n}}{ }^{T} \mathbf{y}_{\mathbf{n}}$, which cancels out with the remaining term in (3.1), minus $\left(\mathbf{X}_{\mathbf{n}}{ }^{T} \mathbf{y}_{\mathbf{n}}\right)^{T}\left(\mathbf{X}_{\mathbf{n}}{ }^{T} \mathbf{X}_{\mathbf{n}}\right)^{-1} \mathbf{X}_{\mathbf{n}}{ }^{T} \mathbf{y}_{\mathbf{n}}$.

Putting these results together yields

$$
\begin{aligned}
\pi\left(\boldsymbol{\beta}, \sigma \mid \mathbf{y}_{\mathbf{n}}\right) & \propto \pi(\sigma) \frac{1}{\sigma^{n}} \exp \left(-\frac{1}{2 \sigma^{2}}\left(\sum_{i=1}^{n} y_{i}^{2}-\left(\mathbf{X}_{\mathbf{n}}{ }^{T} \mathbf{y}_{\mathbf{n}}\right)^{T}\left(\mathbf{X}_{\mathbf{n}}{ }^{T} \mathbf{X}_{\mathbf{n}}\right)^{-1} \mathbf{X}_{\mathbf{n}}{ }^{T} \mathbf{y}_{\mathbf{n}}\right)\right) \\
& \times \exp \left(-\frac{1}{2 \sigma^{2}}\left(\boldsymbol{\beta}-\left(\mathbf{X}_{\mathbf{n}}{ }^{T} \mathbf{X}_{\mathbf{n}}\right)^{-1} \mathbf{X}_{\mathbf{n}}{ }^{T} \mathbf{y}_{\mathbf{n}}\right)^{T} \mathbf{X}_{\mathbf{n}}{ }^{T} \mathbf{X}_{\mathbf{n}}\left(\boldsymbol{\beta}-\left(\mathbf{X}_{\mathbf{n}}{ }^{T} \mathbf{X}_{\mathbf{n}}\right)^{-1} \mathbf{X}_{\mathbf{n}}{ }^{T} \mathbf{y}_{\mathbf{n}}\right)\right) .
\end{aligned}
$$


Therefore,

$$
\boldsymbol{\beta} \mid \sigma, \mathbf{y}_{\mathbf{n}} \sim \mathcal{N}\left(\left(\mathbf{X}_{\mathbf{n}}{ }^{T} \mathbf{X}_{\mathbf{n}}\right)^{-1} \mathbf{X}_{\mathbf{n}}{ }^{T} \mathbf{y}_{\mathbf{n}}, \sigma^{2}\left(\mathbf{X}_{\mathbf{n}}{ }^{T} \mathbf{X}_{\mathbf{n}}\right)^{-1}\right),
$$

and

$$
\begin{aligned}
& \pi\left(\boldsymbol{\beta} \mid \sigma, \mathbf{y}_{\mathbf{n}}\right)=\frac{1}{\sqrt{(2 \pi)^{p}\left|\left(\mathbf{X}_{\mathbf{n}}{ }^{T} \mathbf{X}_{\mathbf{n}} / \sigma\right)^{-1}\right|}} \\
& \times \exp \left(-\frac{1}{2 \sigma^{2}}\left(\boldsymbol{\beta}-\left(\mathbf{X}_{\mathbf{n}}{ }^{T} \mathbf{X}_{\mathbf{n}}\right)^{-1} \mathbf{X}_{\mathbf{n}}{ }^{T} \mathbf{y}_{\mathbf{n}}\right)^{T} \mathbf{X}_{\mathbf{n}}{ }^{T} \mathbf{X}_{\mathbf{n}}\left(\boldsymbol{\beta}-\left(\mathbf{X}_{\mathbf{n}}{ }^{T} \mathbf{X}_{\mathbf{n}}\right)^{-1} \mathbf{X}_{\mathbf{n}}{ }^{T} \mathbf{y}_{\mathbf{n}}\right)\right) \\
& \quad=\frac{1}{\sigma^{p}} \frac{1}{(2 \pi)^{p / 2}}\left|\mathbf{X}_{\mathbf{n}}{ }^{T} \mathbf{X}_{\mathbf{n}}\right|^{1 / 2} \\
& \times \exp \left(-\frac{1}{2 \sigma^{2}}\left(\boldsymbol{\beta}-\left(\mathbf{X}_{\mathbf{n}}{ }^{T} \mathbf{X}_{\mathbf{n}}\right)^{-1} \mathbf{X}_{\mathbf{n}}{ }^{T} \mathbf{y}_{\mathbf{n}}\right)^{T} \mathbf{X}_{\mathbf{n}}{ }^{T} \mathbf{X}_{\mathbf{n}}\left(\boldsymbol{\beta}-\left(\mathbf{X}_{\mathbf{n}}{ }^{T} \mathbf{X}_{\mathbf{n}}\right)^{-1} \mathbf{X}_{\mathbf{n}}{ }^{T} \mathbf{y}_{\mathbf{n}}\right)\right) .
\end{aligned}
$$

Consequently,

$$
\pi\left(\sigma \mid \mathbf{y}_{\mathbf{n}}\right) \propto \pi(\sigma) \frac{1}{\sigma^{n-p}} \exp \left(-\frac{1}{2 \sigma^{2}}\left(\sum_{i=1}^{n} y_{i}^{2}-\left(\mathbf{X}_{\mathbf{n}}^{T} \mathbf{y}_{\mathbf{n}}\right)^{T}\left(\mathbf{X}_{\mathbf{n}}{ }^{T} \mathbf{X}_{\mathbf{n}}\right)^{-1} \mathbf{X}_{\mathbf{n}}{ }^{T} \mathbf{y}_{\mathbf{n}}\right)\right) .
$$

It just remains to prove that

$$
\sum_{i=1}^{n} y_{i}^{2}-\left(\mathbf{X}_{\mathbf{n}}{ }^{T} \mathbf{y}_{\mathbf{n}}\right)^{T}\left(\mathbf{X}_{\mathbf{n}}{ }^{T} \mathbf{X}_{\mathbf{n}}\right)^{-1} \mathbf{X}_{\mathbf{n}}{ }^{T} \mathbf{y}_{\mathbf{n}}=\left\|\mathbf{y}_{\mathbf{n}}-\hat{\mathbf{y}}_{\mathbf{n}}\right\|^{2}
$$

We have

$$
\begin{aligned}
\sum_{i=1}^{n} y_{i}^{2}-\left(\mathbf{X}_{\mathbf{n}}{ }^{T} \mathbf{y}_{\mathbf{n}}\right)^{T}\left(\mathbf{X}_{\mathbf{n}}{ }^{T} \mathbf{X}_{\mathbf{n}}\right)^{-1} \mathbf{X}_{\mathbf{n}}{ }^{T} \mathbf{y}_{\mathbf{n}} & =\mathbf{y}_{\mathbf{n}}{ }^{T} \mathbf{y}_{\mathbf{n}}-\left(\left(\mathbf{X}_{\mathbf{n}}{ }^{T} \mathbf{X}_{\mathbf{n}}\right)^{-1} \mathbf{X}_{\mathbf{n}}{ }^{T} \mathbf{y}_{\mathbf{n}}\right)^{T} \mathbf{X}_{\mathbf{n}}{ }^{T} \mathbf{y}_{\mathbf{n}} \\
& =\mathbf{y}_{\mathbf{n}}{ }^{T} \mathbf{y}_{\mathbf{n}}-\hat{\mathbf{y}}_{\mathbf{n}}^{T} \mathbf{y}_{\mathbf{n}} \\
& =\left(\mathbf{y}_{\mathbf{n}}-\hat{\mathbf{y}}_{\mathbf{n}}\right)^{T}\left(\mathbf{y}_{\mathbf{n}}-\hat{\mathbf{y}}_{\mathbf{n}}^{T}+\hat{\mathbf{y}}_{\mathbf{n}}^{T}\right) \\
& =\left(\mathbf{y}_{\mathbf{n}}-\hat{\mathbf{y}}_{\mathbf{n}}\right)^{T}\left(\mathbf{y}_{\mathbf{n}}-\hat{\mathbf{y}}_{\mathbf{n}}\right)+\left(\mathbf{y}_{\mathbf{n}}-\hat{\mathbf{y}}_{\mathbf{n}}\right)^{T} \hat{\mathbf{y}}_{\mathbf{n}} \\
& =\left(\mathbf{y}_{\mathbf{n}}-\hat{\mathbf{y}}_{\mathbf{n}}\right)^{T}\left(\mathbf{y}_{\mathbf{n}}-\hat{\mathbf{y}}_{\mathbf{n}}\right) .
\end{aligned}
$$

\section{References}

Bunke, O., Milhaud, X., et al. (1998). "Asymptotic behavior of Bayes estimates under possibly incorrect models." Ann. Statist., 26(2): 617-644.

Desgagné, A. (2015). "Robustness to Outliers in Location-Scale Parameter Model using Log-Regularly Varying Distributions." Ann. Statist., 43(4): 1568-1595.

Scheffé, H. (1947). "A Useful Convergence Theorem for Probability Distributions." Ann. Math. Statist., 434-438. 